\title{
Almost Periodic Functions and Their Applications: A Survey of Results and Perspectives
}

\author{
Wei-Shih Du (D), ${ }^{1}$ Marko Kostić $\mathbb{D}^{2},{ }^{2}$ and Manuel Pinto ${ }^{3}{ }^{3}$ \\ ${ }^{1}$ Department of Mathematics, National Kaohsiung Normal University, Kaohsiung 82444, Taiwan \\ ${ }^{2}$ Faculty of Technical Sciences, University of Novi Sad, Trg D. Obradovića 6, Novi Sad 21125, Serbia \\ ${ }^{3}$ Departamento de Matemáticas, Facultad de Ciencias, Universidad de Chile, Santiago de Chile, Chile
}

Correspondence should be addressed to Marko Kostić; marco.s@verat.net

Received 20 February 2021; Revised 15 March 2021; Accepted 17 March 2021; Published 14 April 2021

Academic Editor: Antonio Masiello

Copyright ( 2021 Wei-Shih Du et al. This is an open access article distributed under the Creative Commons Attribution License, which permits unrestricted use, distribution, and reproduction in any medium, provided the original work is properly cited.

The main aim of this survey article is to present several known results about vector-valued almost periodic functions and their applications. We separately consider almost periodic functions depending on one real variable and almost periodic functions depending on two or more real variables. We address several open problems and possibilities for further investigations of almost periodic functions, quoting more than two hundred references about the subject under our consideration.

\section{Introduction}

The class of almost periodic functions was introduced by the Danish mathematician H. Bohr [1] (1925), the younger brother of the Nobel Prize-winning physicist N. Bohr, and later generalized by many others. Let $I=\mathbb{R}$ or $I=[0, \infty)$, let $X$ be a complex Banach space, and let $f: I \longrightarrow X$ be continuous. Given $\varepsilon>0$, we call $\tau>0$ an $\varepsilon$-period for $f(\cdot)$ if and only if

$$
\|f(t+\tau)-f(t)\| \leq \varepsilon, \quad t \in I .
$$

By $\vartheta(f, \varepsilon)$, we denote the set of all $\epsilon$-periods for $f(\cdot)$. We say that $f(\cdot)$ is almost periodic if and only if for each $\epsilon>0$, the set $\vartheta(f, \varepsilon)$ is relatively dense in $[0, \infty)$, which means that there exists $l>0$ such that any subinterval of $[0, \infty)$ of length $l$ meets $\vartheta(f, \varepsilon)$. There are many research monographs concerning the theory of almost periodic functions and their applications; at the very beginning, we would like to cite the important research monograph [2] by Levitan, only.

The class of almost automorphic functions was introduced by the American mathematician Bochner [3]. A continuous function $f: \mathbb{R} \longrightarrow X$ is said to be almost automorphic if and only if for every real sequence $\left(b_{n}\right)$, there exist a subsequence $\left(a_{n}\right)$ of $\left(b_{n}\right)$ and a map $g: \mathbb{R} \longrightarrow X$ such that

$$
\begin{aligned}
& \lim _{n \longrightarrow \infty} f\left(t+a_{n}\right)=g(t), \\
& \lim _{n \longrightarrow \infty} g\left(t-a_{n}\right)=f(t),
\end{aligned}
$$

pointwise for $t \in \mathbb{R}$. Any almost periodic function is almost automorphic, but the converse statement is not true in general (see the research monograph [4] by N'Guerekata for more details). The theories of almost periodic functions and almost automorphic functions are still very active fields of investigations of numerous authors, full of open problems, conjectures, hypotheses, and possibilities for further expansions.

As mentioned in the abstract, this survey article aims to present several known results about vector-valued almost periodic functions and their applications (there is no need to say that it would be very difficult to summarize so many important research results obtained in the theory of almost periodic functions within only one research report, and because of that, we feel it is our duty to say that this survey article does not intend to be exhaustively complete). We divide our further exposition into two individual sections; in Section 1, we analyze the almost periodic functions of one real variable and their applications, while in Section 2, we analyze the almost periodic functions of several real variables and their applications. The material is basically taken from the 
introductory part, notes, and appendices to the second and the third sections of the forthcoming research monograph [5].

\section{Almost Periodic Functions of One Real Variable and Their Applications}

From the application point of view, almost periodic functions of one real variable are much important than the almost periodic functions of two or more real variables. There is enormous literature devoted to the study of almost periodicity in the time variable and the almost automorphy in the time variable of solutions for various kinds of the abstract differential equations of the first order. The notion of an almost periodic strongly continuous semigroup was introduced by Bart and Goldberg in [6], but some particular results concerning the almost periodicity of individual orbits of strongly continuous semigroups were already given by Foias and Zaidman [7], Zhikov [8,9], and Perov and Hai [10]; also, see the survey article [11] by Phóng as well as the reference list of [12] and the articles $[13,14]$ obtained in a collaboration of Phóng and Lyubich. The notion of an asymptotically almost periodic strongly continuous semigroup was introduced by Ruess and Summers [15] in 1986 (see also [16-18]), while the notion of an (asymptotically) Stepanov almost periodic strongly continuous semigroup was introduced by Henríquez [19] in 1990. Concerning the study of the existence and uniqueness of almost periodic solutions of nondegenerate semilinear Cauchy problems, it seems that the fractional powers of operators have been employed for the first time by Bahaj and Sidki in [20]. For the periodic solutions of abstract first-order differential equations, we refer the reader to the research monographs [21] by Burton, [22] by Liu, Guerekata, and Minh, and [23] by Yoshizawa.

The notion of almost periodic cosine operator functions was introduced by Cioranescu [24] and after that received considerable attention of many authors. The existence and uniqueness of almost periodic-type solutions of the (abstract) second-order differential equations have been investigated in many research articles by now, using the theory of cosine operator functions or other methods (see, e.g., [25-33]). For example, Diagana, Hassan, and Messaoudi recently analyzed, in [34], the existence of asymptotically almost periodic mild solutions of the abstract Volterra integrodifferential equation

$$
u^{\prime \prime}(t)+A^{2} u(t)-\int_{-\infty}^{t} g(t-s) A^{2} u(s) \mathrm{d} s=f(t, u(t)), \quad t \geq 0,
$$

accompanied with the initial conditions $u(-t)=u_{0}(t)$ for $t \geq 0$ and $u^{\prime}(0)=u_{1}$. The main strategy used is a transformation of such a system into a first-order linear evolution equation whose solutions are governed by exponentially decaying strongly continuous semigroups; an interesting application was made in the study of Kirchhoff plate equation with infinite memory. Regarding the abstract second-order differential equations in Hilbert spaces, it should also be noted that the existence and uniqueness of periodic solutions for the following equations,

$$
\begin{aligned}
u_{t t}+(A+\gamma I) u(t) & =F(t, u(t)), \quad t \geq 0,(\gamma \in \mathbb{R}), \\
u_{t t}+A^{2} u(t) & =F\left(t, u(t), u^{\prime}(t)\right), \quad t \geq 0, \\
u_{t t}(t)+2 \alpha u_{t}(t)+A u(t) & =g(t)+F(t, u(t)) a, \quad t \geq 0,
\end{aligned}
$$

were analyzed by Strashkraby, Vejvoda (1973), Lovicar (1977), and Masudy (1966), respectively ( $A$ is a positive selfadjoint operator in a Hilbert space $H$ ). For more details about the existence and uniqueness of almost periodic-type solutions of the abstract first-order Cauchy problems and the abstract second-order Cauchy problems, we refer the reader to the reference lists in $[5,12]$. We recall the following problem proposed in [12].

Problem: let a closed multivalued linear operator $\mathscr{A}$ be the integral generator of a bounded $C$-cosine function $(C(t))_{t \geq 0}$. Suppose that $x \in X$ satisfies that the mapping $t \longmapsto C(t) x, t \geq 0$, is asymptotically Stepanov almost periodic. Is it true that the mapping $t \longmapsto C(t) C x, t \geq 0$, is almost periodic?

Chronologically, the study of almost periodic solutions of the abstract Volterra integrodifferential equations was initiated by Prüss in [35], Section 11.4, where the author analyzed the almost periodic solutions, Stepanov almost periodic solutions, and asymptotically almost periodic solutions of the following abstract integrodifferential equation:

$$
\begin{array}{r}
u^{\prime}(t)=\int_{0}^{\infty} A_{0}(s) u^{\prime}(t-s) \mathrm{d} s+\int_{0}^{\infty} \mathrm{d} A_{1}(s) u(t-s)+f(t), \\
t \in \mathbb{R} .
\end{array}
$$

Here, $A_{0} \in L^{1}([0, \infty): L(Y, X)), t \longmapsto A_{1}(t) \in L(Y, X)$, $t \geq 0$, is locally of bounded variation, and $X$ and $Y$ are Banach spaces such that $Y$ is densely and continuously embedded into $X$. Almost immediately after that, $\mathrm{Vu}[36]$ investigated the almost periodicity of the abstract Cauchy problem

$$
u^{\prime}(t)=A u(t)+\int_{0}^{\infty} \mathrm{d} B u(\tau) u(t-\tau)+f(t), \quad t \in \mathbb{R},
$$

where $A$ is a closed linear operator acting on a Banach space $X,(B(t))_{t \geq 0}$ is a family of closed linear operators on $X$, and $f: \mathbb{R} \longrightarrow X$ is continuous.

It is very difficult and unpleasant to say precisely who was the first to study the almost periodic solutions of the abstract fractional differential equations. Recently, $\mathrm{Mu}$, Zhoa, and Peng [37] investigated the periodic solutions and $S$-asymptotically periodic solutions to fractional evolution equation $D_{t,+}^{\gamma} u(t)=-A u(t)+g(t), t \in \mathbb{R}$, and its semilinear analogue $D_{t+,}^{\gamma} u(t)=-A u(t)+g(t, u(t)), t \in \mathbb{R}$, where $D_{t,+}^{\gamma}$ denotes the Weyl-Liouville fractional derivative of order $\gamma \in(0,1), A$ is the infinitesimal generator of an exponentially decaying strongly continuous semigroup of operators, and $g: \mathbb{R} \times X \longrightarrow X$ satisfies certain assumptions (also, see the article [38] by Agarwal, Andrade, and Cuevas as 
well as the recent articles [39] by Bedi, Kumar, Abdeljawad, and Khan and [40] by Brindle and Guérékata, where the authors analyzed $S$-asymptotically $\omega$-periodic mild solutions for fractional differential equations with Hilfer derivatives and Riemann-Liouville derivatives). Later, Kostić extended the results of $\mathrm{Mu}$, Zhoa, and Peng to the abstract fractional differential inclusion $D_{t,+}^{\gamma} u(t) \in-\mathscr{A} u(t)+g(t), t \in \mathbb{R}$, and its semilinear analogue

$$
D_{t,+}^{\gamma} u(t) \in-\mathscr{A} u(t)+g(t, u(t)), \quad t \in \mathbb{R},
$$

where $\mathscr{A}$ is a closed multivalued linear operator satisfying condition (P); here, we follow the terminology employed in [41], where we have obeyed the multivalued approach to the abstract degenerate Volterra integrodifferential equations. (P) There exist finite constants $c, M>0$ and $\beta \in(0,1]$ such that $\Psi:=\Psi_{c}:=\{\lambda \in \mathbb{C}: \mathfrak{R} \lambda \geq-c(|\Im \lambda|+1)\} \subseteq \rho(\mathscr{A})$ and $\|R(\lambda: \mathscr{A})\| \leq M(1+|\lambda|)^{-\beta}, \lambda \in \Psi$. The obtained results enable one to consider the almost periodic-type solutions of the following fractional Poisson heat equations,

$$
\begin{aligned}
& \begin{cases}\frac{\partial}{\partial t}[m(x) v(t, x)]=(\Delta-b) v(t, x)+f(t, m(x) v(t, x)), & t \in \mathbb{R}, x \in \Omega, \\
v(t, x)=0, & (t, x) \in[0, \infty) \times \partial \Omega,\end{cases} \\
& \begin{cases}\mathbf{D}_{t}^{\gamma}[m(x) v(t, x)]=\Delta v(t, x)+b v(t, x), & t \geq 0, x \in \Omega, \\
v(t, x)=0, & (t, x) \in[0, \infty) \times \partial \Omega, \\
m(x) v(0, x)=u_{0}(x), & x \in \Omega,\end{cases}
\end{aligned}
$$

and the following fractional semilinear equation with higher-order differential operators in the Hölder space $X=C^{\alpha}(\bar{\Omega})$ :

$$
\begin{cases}\mathbf{D}_{t}^{\gamma} u(t, x)=-\sum_{|\beta| \leq 2 m} a_{\beta}(t, x) D^{\beta} u(t, x)-\sigma u(t, x)+f(t, u(t, x)), & t \geq 0, x \in \Omega, \\ u(0, x)=u_{0}(x), & x \in \Omega .\end{cases}
$$

See [12] for more details. Let us also recall that Ponce [42] investigated the bounded mild solutions of the following nondegenerate fractional integrodifferential equation:

$$
D_{t,+}^{\gamma} u(t)=A u(t)+\int_{-\infty}^{t} a(t-s) A u(s) \mathrm{d} s+f(t, u(t)), \quad t \in \mathbb{R},
$$

where $A$ is a closed linear operator, $a \in L^{1}([0, \infty))$ is a scalar-valued kernel, and $f(\cdot, \cdot)$ satisfies some Lipschitz-type conditions. In particular, almost periodic solutions of (10) have been analyzed. Abbas, Kavitha, and Murugesu recently analyzed Stepanov-like (weighted) pseudo-almost automorphic solutions to the following fractional-order abstract integrodifferential equation:

$$
D_{t}^{\alpha} u(t)=A u(t)+D_{t}^{\alpha-1} f(t, u(t), K u(t)), \quad t \in \mathbb{R},
$$

where

$$
K u(t)=\int_{-\infty}^{t} k(t-s) h(s, u(s)) \mathrm{d} s, \quad t \in \mathbb{R},
$$

$1<\alpha<2, A$ is a sectorial operator with the domain and range in $X$, of negative sectorial type $\omega<0$, the function $k(t)$ is exponentially decaying, and the functions $f: \mathbb{R} \times X \times X \longrightarrow X$ and $h: \mathbb{R} \times X \longrightarrow X$ are Stepanov-like weighted pseudo-almost automorphic in time for each fixed element of $X \times X$ and $X$, respectively, satisfying some extra conditions [43]. For more details about almost periodic-type solutions of the abstract fractional differential equations, see the reference list of [12] and the articles [44-48].

As mentioned from the above, many results concerning the existence and uniqueness of almost periodic-type solutions and almost automorphic-type solutions to the abstract (semilinear) fractional nondegenerate differential equations have been given recently by numerous authors. In almost all these results (in the linear setting, the quite exceptional are some examples and results presented by Zaidman ([49], Examples 4, 5, 7, and 8; pp. 32-34), which have been employed by many authors so far, for various purposes), the basic key is to investigate the invariance of certain kinds of generalized almost periodicity and generalized almost automorphicity under the actions of the infinite convolution products 


$$
t \longmapsto \int_{-\infty}^{t} R(t-s) f(s) \mathrm{d} s, \quad t \in \mathbb{R}
$$

and

$$
t \longmapsto \int_{0}^{\infty} R(t-s) f(s) \mathrm{d} s, \quad t \geq 0
$$

Here, it is commonly assumed that $(R(t))_{t \geq 0} \subseteq L(X, Y)$ is a nondegenerate strongly continuous operator family between the Banach spaces $X$ and $Y$ which exponentially or, at least, polynomially decays as $t \longrightarrow+\infty$. In [12], we have investigated the case in which $(R(t))_{t>0} \subseteq L(X, Y)$ is a degenerate strongly continuous operator family which decays exponentially or polynomially as $t \longrightarrow+\infty$, but we have allowed $(R(t))_{t>0}$ to have a removable singularity at zero, by that we basically mean that there exists a number $\zeta \in(0,1)$ such that the operator family $\left(t^{\zeta} R(t)\right)_{t \geq 0}$ is well defined and strongly continuous at the point $t=0$. The integral generator of $(R(t))_{t \geq 0}$ is not single-valued any longer, and this is the main reason why we have employed the multivalued linear approach to the abstract degenerate integrodifferential equations in [12]. The well-posedness of the abstract degenerate Cauchy problem,

$$
B u(t)=f(t)+\int_{0}^{t} a(t-s) A u(s) \mathrm{d} s, \quad t \in[0, \tau),
$$

where $0<\tau \leq \infty, t \longmapsto f(t), t \in[0, \tau)$ is a continuous mapping, $a \in L_{\text {loc }}^{1}([0, \tau))$, and $A, B$ are closed linear operators, has been thoroughly analyzed in the monograph [41].

We will say just a few words about periodic solutions of the abstract degenerate Volterra integrodifferential equations. In [50], Barbu and Favini analyzed the 1-periodic solutions of the abstract degenerate differential equation $(\mathrm{d} / \mathrm{d} t)(B u(t))=A u(t), t \geq 0$, accompanied with the initial condition $(B u)(0)=(B u)(1)$, by using Grisvard's sum of operators method and some results from the investigation of Prüss [51] in the nondegenerate case. The authors reduced the above problem to $v^{\prime}(t) \in \mathscr{A} v(t), t \geq 0, v(0)=v(1)$, where the multivalued linear operator $\mathscr{A}$ is given by $\mathscr{A}=A B^{-1}$. The main problem is whether the inclusion $1 \in \rho(\mathscr{A})$ holds or not; recall that Prüss [51] proved that $1 \in \rho(A)$ if and only if $2 \pi i \mathbb{Z} \subseteq \rho(A)$ and $\sup \left(\left\{\left\|(2 \pi i n-A)^{-1}\right\|: n \in \mathbb{Z}\right\}\right)<\infty$, provided that $A$ generates a nondegenerate strongly continuous semigroup. Applications are given to the Poisson heat equation in $H^{-1}(\Omega)$ and $L^{2}(\Omega)$, as well as to some systems of ordinary differential equations. On the contrary, Lizama and Ponce [52] analyzed the existence of $2 \pi$-periodic solutions to the following abstract inhomogeneous linear equation:

$$
\frac{\mathrm{d}}{\mathrm{d} t}(B u(t))=A u(t)+\int_{-\infty}^{t} a(t-s) A u(s) \mathrm{d} s+f(t), \quad t \geq 0,
$$

subjected with the initial condition $(B u)(0)=(B u)(2 \pi)$. The authors also considered the maximal regularity of $(16)$ in periodic Besov, Triebel-Lizorkin, and Lebesgue vector-valued function spaces.
Concerning the classical theory of partial differential equations with integer-order derivatives, we would like to recommend for the reader the references and works quoted in the introductory part of the fourth section of the monograph [53] by Ptashnic, where the following have been especially emphasized:

(1) The $\omega$-periodic solutions in time for the linear wave equation and the following weakly nonlinear wave equation $\quad u_{t t}(t, x)-u_{x x}(t, x)=\varepsilon f\left(t, x, u, u_{t}, u_{x}\right.$, $\varepsilon), \quad t \geq 0,0 \leq x \leq \pi$, accompanied with the boundary conditions $u(t, 0)=u(t, \pi)=0$, were analyzed by Vejvoda [54] in 1964 ( $\varepsilon>0$ is a sufficiently small real parameter). If $\omega \in 2 \pi \mathbb{Q}$ and $\omega>0$, then the existence of $\omega$-periodic solutions for both classes of wave equations was proved; on the contrary, if $\omega \notin 2 \pi \mathbb{Q}$ and $\omega>0$, then the situation is much more complicated, and the author proved the existence of $\omega$-periodic solutions for a corresponding linear wave equation, only, provided that $\omega=2 \pi \alpha$ and there exist positive real numbers $c>0$ and $\gamma>0$ such that $|\alpha-(m / k)|>\left(c / k^{\gamma}\right)$.

Only one year later, in 1965, Gavlova investigated the existence and uniqueness of periodic solutions for the following weakly nonlinear telegraph equation: $u_{t t}-u_{x x}+2 a u_{t}+2 b u_{x}+c u=h(t, x)+\varepsilon f\left(t, u, \quad u_{t}\right.$, $\left.u_{x}, \varepsilon\right)$, accompanied with the boundary conditions $u(t, 0)=u(t, \pi)=0$, where $a, b, c \in \mathbb{R}$ are certain constants and $\varepsilon>0$ is a sufficiently small real parameter.

(2) In 1972, Azis and Gorak investigated the existence and uniqueness of periodic solutions in the time variable and space variable for the following quasilinear hyperbolic second-order equation $u_{x y}+a(x, y) u_{x}+b(x, y) u_{y}+c(x, y) u=f(x, y, u$, $\left.u_{x}, u_{y}\right)$; in 1971, Krylovoi and Vejvoda investigated the existence and uniqueness of $\omega$-periodic solutions in the time variable for the following equation: $u_{t t}+$ $u_{x x x x}=g(t, x)+\varepsilon f\left(t, x, u, u_{x}, u_{x x}, u_{t}, \varepsilon\right)$, accompanied with the boundary conditions $u(t, 0)=u(t, 2 \pi)=u_{x x}(t, 0)=u_{x x}(t, \pi)=0$.

Six years later, in 1977, Kopachkovoi and Vejvoda analyzed the existence and uniqueness of $\omega$-periodic solutions in the time variable for the following nonlinear equation: $u_{t t}+u_{x x x x}-\varepsilon u_{x x} \int_{0}^{\pi} u^{2}(x, \xi) \mathrm{d} \xi=g(t, x)+\varepsilon^{2} F(u)(t, x)$, which appears in the study of beam vibrations with the effect of elongation. Also, see the important research monograph [55] by Vejvoda (with Herrmann and Lovicar as contributors).

Furthermore, the Bohr almost periodic solutions to boundary value problems for systems of partial differential equations that arise in solving certain problems for inhomogeneous media have been investigated in the research articles [56] by Berselli and Bisconti, [57] by Berselli and Romito, and [58] by Vetchanin and Mikishanina. Concerning the existence and uniqueness of Bohr almost periodic solutions of the Navier-Stokes-type equations, the reader may consult the reference list of [5]. 
The study of differential equations with discontinuous arguments was initiated by Myshkis [59] in 1977. The analysis of asymptotically antiperiodic solutions for nonlinear differential first-order equations with piecewise constant argument carried out by Dimbour and Valmorin [60] has recently been reconsidered and extended for asymptotically Bloch periodic solutions for nonlinear fractional differential inclusions with a piecewise constant argument by Kostić and Velinov in [61]. We have analyzed the following fractional differential Cauchy inclusion with a piecewise constant argument:

$$
\mathbf{D}_{t}^{\gamma} u(t) \in \mathscr{A} u(t)+A_{0} u(\lfloor t\rfloor)+g(t, u(\lfloor t\rfloor)), t>0 ; \quad u(0)=u_{0},
$$

where $\mathscr{A}$ is a multivalued linear operator satisfying certain assumptions, $A_{0} \in L(X), g:[0, \infty) \times X \longrightarrow X$ is a given function, and $\mathbf{D}_{t}^{\gamma} u(t)$ denotes the Caputo fractional derivative of order $\gamma$, taken in a weak sense. It is also worth noting that Chávez, Castillo, and Pinto [62] analyzed the existence of a unique almost automorphic solution for the following differential equation with a piecewise constant argument:

$$
y^{\prime}(t)=A(t) y(t)+B(t) y(\lfloor t\rfloor)+f(t, y(t), y(\lfloor t\rfloor)), \quad t \in \mathbb{R},
$$

where $A(t)$ and $B(t)$ are almost automorphic $p \times p$ complex matrices and $f: \mathbb{R} \times \mathbb{C}^{p} \times \mathbb{C}^{p} \longrightarrow \mathbb{C}^{p}$ is an almost automorphic function satisfying a condition of Lipschitz type. The study carried out in [62] leans heavily on the use of results on discontinuous almost automorphic functions, exponential dichotomies, and the Banach fixed-point theorem. The almost periodic solutions of (18) were considered for the first time by Yuan and Hong in [63]; for more details about differential equations with a piecewise constant argument (DEPCA), the reader may consult articles [64] by Cooke and Wiener, [65] by Shah and Wiener, and [66] by Wiener, as well as articles [67-73], the list of publication of Pinto (https://www.zbmath.org/?q=ai(percent/sign) 3Apinto.manuel), and the list of references cited therein.

There is a vast amount of articles in the existing literature which consider almost automorphic-type solutions for various classes of integrodifferential equations. Let us only mention our analysis (the joint work of the second-named author with Prof. Guérékata [74]) of the following abstract multiterm fractional differential inclusion:

$$
\begin{aligned}
& \mathbf{D}_{t}^{\alpha_{n}} u(t)+\sum_{i=1}^{n-1} A_{i} \mathbf{D}_{t}^{\alpha_{i}} u(t) \in \mathscr{A} \mathbf{D}_{t}^{\alpha} u(t)+f(t), \quad t \geq 0, \\
& u^{(k)}(0)=u_{k}, k=0, \ldots,\left\lceil\alpha_{n}\right\rceil-1,
\end{aligned}
$$

where $n \in \mathbb{N} \backslash\{1\}, A_{1}, \ldots, A_{n-1}$ are bounded linear operators on a Banach space $X, \mathscr{A}$ is a closed multivalued linear operator on $X, 0 \leq \alpha_{1}<\cdots<\alpha_{n}, 0 \leq \alpha<\alpha_{n}, f(\cdot)$ is an $X$-valued function, and $\mathbf{D}_{t}^{\alpha}$ denotes the Caputo fractional derivative of order $\alpha$. Many excellent examples have been presented in monograph [75] by Diagana; also, see the following monographs:
(1) [76] by Amerio and Prouse for almost periodic solutions of functional equations

(2) [77] by Argabright and de Lamadrid for almost periodic measures

(3) $[78,79]$ by Baake and Grimm for applications of almost periodic functions in crystallography

(4) [80] by Bezandry and Diagana for almost periodic solutions of stochastic differential equations

(5) [81] by Böttcher, Karlovich, and Spitkovsky for factorization of almost periodic matrix functions (cf. also article [82] by Böttcher for the issues regarding the corona theorem for almost periodic functions of several real variables and articles [83] by Boggiatto, Ferández, and Galbis and [84] by Kim for issues concerning Gabor systems and almost periodic functions)

(6) [85] by Chang, Guerekata, and Ponce for Bloch $(p, k)$-periodic functions, antiperiodic functions, and their applications

(7) [86] by Cheban for asymptotically almost periodic solutions of linear and nonlinear equations

(8) [87] by Emel'yanov for weakly almost periodic $C_{0}$-semigroups

(9) [88] by Hino, Naito, Minh, and Shin and [89] by Guérékata for spectral analysis of almost periodic functions and Massera-type theorems [90]

(10) [91] by Hsu for weakly almost periodic functions

(11) [92] by Stamov for almost periodic solutions of impulsive differential equations (see also research monographs [93] by Bainov and Simeonov, [94] by Perestyuk, Plotnikov, Somoilenko, and Skripnik, [95] by Stamova and Stamov, and [96] by Song, Gno, and Shi for more details on the subject)

Concerning the existence and uniqueness of almost periodic-type solutions of inhomogeneous evolution equations of first order, the notions of hyperbolic evolution systems and Green's functions are incredible important; for more details on the subject, we refer the reader to Acquistapace [97], Acquistapace and Terreni [98], Chang and Chen [99], Diagana [75], Khalil [100], Schnaubelt [101], Zhikov [102, 103], and the list of references in [12]. The almost periodic- and almost automorphic-type solutions of the abstract Cauchy problems,

$$
\begin{array}{ll}
u^{\prime}(t)=A(t) u(t)+f(t), & t \in \mathbb{R}, \\
u^{\prime}(t)=A(t) u(t)+f(t), & t>0 ; u(0)=x,
\end{array}
$$

and their semilinear analogues have been investigated in a great number of research papers. Without going into full details, we will only refer the readers to research monographs [75] by Diagana and [12] by Kostić, articles [104] by Baroun, Maniar, and Schnaubelt and [105] by Baroun, Ezzinbi, Khalil, and Maniar, and the list of references therein. Concerning the applications of evolution systems in the theory of the second-order nonautonomous differential 
equations, mention should be made of paper [106] by Zakora.

The almost periodic and almost automorphic functions on time scales and their applications to the abstract Volterra integrodifferential equations have recently been considered by numerous mathematicians (for time-scale calculus, we warmly recommend monograph [107] by Bochner and Peterson). It would be really troublesome to quote here all relevant references concerning the almost periodic traveling wave solutions and the almost automorphic traveling wave solutions for various classes of nonlinear partial differential equations. For more details about the above problematic, we refer the reader to the references cited in [5].

The definitions and basic properties of $(\omega, c)$-periodic and $(\omega, c)$-pseudo-periodic functions were introduced and analyzed by Alvarez, Gómez, and Pinto in [108, 109], motivated by some known results regarding the qualitative properties of the solution to Mathieu's linear differential equation $y^{\prime \prime}(t)+[a-2 q \cos 2 t] y(t)=0$, arising in modeling of railroad rails and seasonally forced population dynamics $(\omega>0$ and $c \in \mathbb{C} \backslash\{0\})$. The linear delayed equations can have $(\omega, c)$-periodic solutions as well (see, e.g., [109], Example 2.5). The notions of antiperiodicity and Bloch periodicity are special cases of the notion of an $(\omega, c)$-periodicity, which has also been analyzed in [110].

The authors of [109] analyzed the existence and uniqueness of mild $(\omega, c)$-periodic solutions to abstract semilinear integrodifferential equation (10). Furthermore, Alvarez, Castillo, and Pinto analyzed in [108] the existence and uniqueness of mild $(\omega, c)$-pseudo-periodic solutions to the abstract semilinear differential equation of the first order:

$$
u^{\prime}(t)=A u(t)+f(t, u(t)), \quad t \in \mathbb{R},
$$

where $A$ generates a strongly continuous semigroup. The authors proved the existence of positive $(\omega, c)$-pseudo-periodic solutions to the Lasota-Wazewska equation with $(\omega, c)$-pseudo-periodic coefficients:

$$
y^{\prime}(t)=-\delta y(t)+h(t) e^{-a(t) y(t-\tau)}, \quad t \geq 0 .
$$

This equation describes the survival of red blood cells in blood of an animal (see, e.g., Wazewska-Czyzewska and Lasota [111]). Concerning the applications to time-varying impulsive differential equations, mention should be made of article [112] by Wang, Ren, and Zhou; also, cf. article [113] by Mophou, Guérékata, and Milce and article [114] by Li, Wang, and Fečkan. For further information about (weighted) pseudo-almost periodic solutions and (weighted) pseudo-almost automorphic solutions of various types of abstract Volterra integrodifferential equations, we refer the reader to [115-122] and [123-130].

Before we explain the main results and applications of multidimensional-type functions, we will single out a few important topics for our readers.

Almost periodic functions of complex variables: the theory of almost periodic functions of one complex variable, initiated already by Bohr in the third part of [1], is still very popular and attracts the attention of many mathematicians (see, e.g., [131-134]). Suppose that $-\infty \leq \alpha<\beta \leq+\infty$ and the function $f: \Omega \equiv\{z \in \mathbb{C}: \alpha<\Re z<\beta\} \longrightarrow X$ is analytic. Then, we say that $f(\cdot)$ is almost periodic if and only if for any $\varepsilon>0$ and every reduced strip $\left\{z \in \mathbb{C}: \alpha^{\prime}<\mathfrak{R} z<\beta^{\prime}\right\}$, where $\alpha<\alpha^{\prime}<\beta^{\prime}<\beta$, there exists a number $l>0$ such that each subinterval of length $l$ of $\mathbb{R}$ contains a number $\tau$ satisfying the inequality

$$
\|f(z+i \tau)-f(z)\| \leq \varepsilon, \quad \text { for } \alpha^{\prime}<\Re z<\beta^{\prime} .
$$

In particular, this definition implies that, for any fixed $\sigma \in(\alpha, \beta)$, the function $f_{\sigma}(t):=f(\sigma+i t), t \in \mathbb{R}$, is almost periodic. Moreover, the definition implies that the almost periodicity should be uniform on various straight lines, with the meaning being clear. The Fourier series of these functions can be obtained from a certain exponential series with complex coefficients; the associated series is called the Dirichlet series of $f(\cdot)$. As for the functions of one real variable, Bohr's notion of almost periodicity of $f(\cdot)$ in a vertical strip $\Omega$ is equivalent to the relative compactness of the set of its vertical translates, $\{f(\cdot+i h): h \in \mathbb{R}\}$, with the topology of the uniform convergence on reduced strips. Mean motions and zeros of generalized almost periodic analytic functions have been analyzed by Borchsenius and Jessen in [135], where the reader can find several important applications to the Riemann zeta function (also, see [136] and the references therein for further information about applications of results from the theory of almost periodic analytic functions to the Riemann zeta function). For more details about subharmonic almost periodic functions and holomorphic almost periodic functions, we refer the reader to $[131,137-140]$ and references cited therein.

$C^{(n)}$-almost periodic functions: the notion of $C^{(n)}$-almost periodicity was introduced by Adamczak [141] in 1997 and later received great attention of many other authors. In this article, we will only say a few words about generalized $C^{(n)}$-almost periodic functions and possibilities for further expansions. Several different classes of Stepanov-like $C^{(n)}$-pseudo-almost automorphic functions have been analyzed by Diagana, Nelson, and N'Guérékata in [142]. For example, let $1 \leq p<\infty$, let $n \in \mathbb{N}$, and let $f \in L_{\text {loc }}^{p}(I: X)$. Then, we say that (see [5] for the notion)

(i) the function $f(\cdot)$ is Stepanov- $p$ - $C^{(n)}$-almost periodic, $f \in C^{(n)}-\operatorname{APS}^{p}(I: X)$ for short, if and only if for each $k=0,1, \ldots, n$, we have that $f^{(k)} \in \operatorname{APS}^{p}(I: X)$.

(ii) the function $f \in L_{\mathrm{loc}}^{p}([0, \infty): X)$ is asymptotically Stepanov- $p$ - $C^{(n)}$-almost periodic, $f \in C^{(n)}-\operatorname{AAPS}^{p}([0, \infty): X)$ for short, if and only if for each $k=0,1, \ldots, n$, we have that $f^{(k)} \in \operatorname{AAPS}^{p}([0, \infty): X)$. The following definitions have been analyzed in [12].

(iii) the function $f(\cdot)$ is equi-Weyl- $p$ - $C^{(n)}$-almost periodic, $f \in e-C^{(n)}-W_{a p}^{p}(I: X)$ for short, if and only if for each $k=0,1, \ldots, n$, we have that $f^{(k)} \in e-W_{a p}^{p}(I: X)$.

(iv) the function $f(\cdot)$ is Weyl- $p$ - $C^{(n)}$-almost periodic, $f \in C^{(n)}-W_{a p}^{p}(I: X)$ for short, if and only if for each $k=0,1, \ldots, n$, we have that $f^{(k)} \in W_{a p}^{p}(I: X)$. 
(v) the function $f(\cdot)$ is Besicovitch-Doss- $p-C^{(n)}$-almost periodic, $f \in C^{(n)}-\mathrm{B}^{p}(I: X)$ for short, if and only if for each $k=0,1, \ldots, n$, we have that $f^{(k)} \in \mathrm{B}^{p}(I: X)$.

Using the same idea, we can introduce and analyze a great number of $C^{(n)}$-almost automorphic function spaces [12]. For example, the function

$$
f(t)=\sum_{n=1}^{\infty} \frac{\sin n t}{n^{4}}, \quad t \in \mathbb{R}
$$

is $C^{(2)}$-almost periodic but not $C^{(3)}$-almost automorphic. Furthermore, for any real-valued function $g \in C^{(3)}-A A(\mathbb{R}: \mathbb{C})$ satisfying $\inf _{t \in \mathbb{R}} g^{\prime \prime} \prime(t)>0$, we have that the function

$$
f(t)=\sum_{n=1}^{\infty} \frac{g(n t)}{n^{4}}, \quad t \in \mathbb{R},
$$

belongs to the space $C^{(2)}-\mathrm{AAS}^{1}(\mathbb{R}: \mathbb{C}) \backslash C^{(3)}-\mathrm{AAS}^{1}(\mathbb{R}: \mathbb{C})$; see, e.g., [142], Example 2.23. It is clear that we can slightly generalize the notion of all the aforementioned function spaces by using the definitions and results from the theory of $L^{p(x)}$-spaces.

Before proceeding further, we also want to mention research articles [2, 124, 143-147] by the second-named author as well as to recall the following question proposed in [12]: is it true that the classes of Besicovitch- $p$-almost periodic functions and Besicovitch-Doss- $p$-almost periodic functions coincide in vector-valued case $(1 \leq p<\infty)$ ?

Nemytskii operators between Stepanov almost periodic function spaces: let $p$ and $q$ be two real numbers belonging to the interval $[1, \infty)$, and let $T>0$. It is said that $f:(0, T) \times$ $X \longrightarrow Y$ is a Carathéodory function if and only if the following holds:

(i) The mapping $t \longmapsto f(t, x), t \in(0, T)$, is measurable for any fixed element $x \in X$

(ii) For a.e. $t \in(0, T)$, the function $f(t, \cdot)$ is continuous from $X$ and $Y$

Now, consider the Nemytskii operator $\mathcal{N}_{f}: L^{p}((0, T): X) \longrightarrow L^{q}((0, T): Y)$ by

$$
\left[\mathcal{N}_{f}(\omega)\right](t):=f(t, \omega(t)), \quad t \in(0, T), \omega \in L^{p}((0, T): X) .
$$

The well-known result of Lucchetti and Patrone ([148], Theorem 3.1) states that the Nemytskii operator is well defined between these spaces if and only if there exist $a>0$ and $b \in L^{p}((0, T))$ such that, for all $x \in X$ and a.e. $t \in(0, T)$, we have

$$
\|f(t, x)\| \leq a\|x\|^{(p / q)}+b(t) .
$$

In this case, the Nemytskii operator is continuous.

Concerning the Nemytskii operator between the spaces of almost periodic functions $A P(\mathbb{R}: X)$ and $A P(\mathbb{R}: Y)$, it should be noted that we have the equivalence of the following statements (see, e.g. Blot, Cieutat, Guérékata, and Pennequin [149]): (i) The Nemytskii operator $\mathcal{N}_{f}: A P(\mathbb{R}: X) \longrightarrow$ $A P(\mathbb{R}: Y)$ is continuous.

(ii) For each compact set $K \subseteq X$ and for each $\varepsilon>0$, the set

$\left\{\tau \in \mathbb{R}: \sup _{t \in \mathbb{R}} \sup _{x \in K}\|f(t+\tau)-f(t, x)\| \leq \varepsilon\right\}$,

is relatively dense in $\mathbb{R}$.

(iii) For all $x \in X, f(\cdot, x) \in A P(\mathbb{R}: Y)$, and for each compact set $K \subseteq X$ and for each $\varepsilon>0$, there exists $\delta>0$ such that, for each $x_{1}, x_{2} \in K$ and for each $t \in \mathbb{R}$, we have the implication: $\left\|x_{1}-x_{2}\right\| \leq$ $\delta \Longrightarrow\left\|f\left(t, x_{1}\right)-f\left(t, x_{2}\right)\right\| \leq \varepsilon$.

A similar statement holds for the continuity of the Nemytskii operator between the spaces of almost automorphic functions $A A(\mathbb{R}: X)$ and $A A(\mathbb{R}: Y)$; see, e.g., the recent paper ([150], Theorem 2.3) by Cieutat. Several necessary and sufficient conditions clarifying the continuity of Nemytskii operators between almost periodic and almost automorphic spaces in the sense of Stepanov approach can be found in [150], Section 4.

Geometric properties of generalized almost periodic function spaces of Orlicz type: in his fundamental paper [151], Hillmann investigated the Besicovitch-Orlicz spaces of almost periodic functions. After that, numerous mathematicians working in the field of almost periodic functions have investigated the geometric properties of generalized almost periodic function spaces of Orlicz type. Here, we will describe the results of Morsli and Smaali established in [152] and the results of Bedouhene, Djabri, and Boulahia established in [153], only; for more details on the subject, we refer the reader to the list of references quoted in these papers and [5].

Assume that the function $\varphi: \mathbb{R} \times[0, \infty) \longrightarrow[0, \infty)$ satisfies the following conditions:

(i) For every $t \in \mathbb{R}$, we have $\varphi(t, 0)=0$

(ii) For every $t \in \mathbb{R}$, the mapping $u \longmapsto \varphi(t, u), u \geq 0$, is convex

(iii) $\varphi(t+1, u)=\varphi(t, u)$ for all $t \in \mathbb{R}$ and $u \geq 0$

(iv) For every $u>0$, we have $\inf _{t \in \mathbb{R}} \varphi(t, u)=\phi(u)>0$

If $f: \mathbb{R} \longrightarrow[0,+\infty]$ is a measurable function, then it is well known that the function

$$
f \longmapsto \rho_{\varphi}(f):=\lim \sup _{t \longrightarrow+\infty} \frac{1}{2 t} \int_{-t}^{t} \varphi(t|f(t)|) \mathrm{d} t, \quad f \in M(\mathbb{R}),
$$

is convex and pseudo-modular.

In [152], the authors defined the Besicovitch-Musielak-Orlicz space associated to $\varphi(\cdot, \cdot)$ by

$$
B^{\varphi}(\mathbb{R}):=\left\{f \in M(\mathbb{R}): \lim _{\alpha \longrightarrow 0+} \rho_{\varphi}(\alpha f)=0\right\} .
$$

We have

$$
B^{\varphi}(\mathbb{R})=\left\{f \in M(\mathbb{R}):(\exists \alpha>0), \quad \rho_{\varphi}(\alpha f)<\infty\right\} .
$$

The space $B^{\varphi}(\mathbb{R})$ is equipped with the pseudo-norm $\|f\|_{\varphi}:=\left\{k>0: \rho_{\varphi}(f / k) \leq 1\right\}$. 
The authors introduced two different types of Besicovitch-Musielak-Orlicz spaces of almost periodic functions, $\widetilde{B}_{\text {a.p. }}^{\varphi}(\mathbb{R})$ and $B_{\text {a.p. }}^{\varphi}(\mathbb{R})$, as follows: A function $f: \mathbb{R} \longrightarrow \mathbb{C}$ is said to belong to the space $B_{\text {a.p. }}^{\varphi}(\mathbb{R})$, resp. $\widetilde{B}_{a . p .}^{\varphi}(\mathbb{R})$, if and only if there exists a sequence $\left(f_{n}\right)$ of trigonometric polynomials such that, for every $k>0$, resp. there exists $k>0$ such that $\lim _{n \longrightarrow+\infty} \rho_{\varphi}\left(k\left(f_{n}-f\right)\right)=0$. Then, we clearly have $B_{\text {a.p. }}^{\varphi}(\mathbb{R}) \subseteq \widetilde{B}_{\text {a.p. }}^{\varphi}(\mathbb{R}) \subseteq B^{\varphi}(\mathbb{R})$.

If $\varphi(t,|x|)=|x|$, then by $B_{\text {a.p. }}^{1}(\mathbb{R}), \widetilde{B}_{\text {a.p. }}^{1}(\mathbb{R})$, and $B^{1}(\mathbb{R})$, we denote the respective spaces.

Let us recall that a function $\varphi: \mathbb{R} \times[0, \infty) \longrightarrow[0, \infty)$ is strictly convex if and only if $\varphi(t, \lambda u+(1-\lambda) v)<\lambda \varphi(t, u)+$ $(1-\lambda) \varphi(t, v)$ for a.e. $t \in \mathbb{R}$ and for all $\lambda \in(0,1)$, $0 \leq u<v<\infty$. On the contrary, a normed linear space $(E, \| \cdot$ $\|)$ is said to be strictly convex if and only if

$$
\left\|\frac{x+y}{2}\right\|<1, \quad \text { provided that }\|x\|=\|y\|=1 \text { and } x \neq y .
$$

It is said that the function $\varphi(\cdot, \cdot)$ satisfies the $\Delta_{2}$-condition if and only if there exist a number $k>1$ and a measurable nonnegative function $h(\cdot)$ such that $\rho_{\varphi}(h)<\infty$ and $\varphi(t, 2 u) \leq k \varphi(t, u)$ for almost all $t \in \mathbb{R}$ and all $u \geq h(t)$.

Let $f \in B_{\text {a.p. }}^{\varphi}(\mathbb{R})$. Then, due to [152], Proposition 1 , we have $\varphi(\cdot,|f(\cdot)|) \in B_{\text {a.p. }}^{1}(\mathbb{R})$ so that the limit $\lim _{T \longrightarrow+\infty} 1 / 2 T \int_{-T}^{T} \varphi(t,|f(t)|) \mathrm{d} t$ always exists and is finite. The main result of paper is [152], Theorem 1 , which states that the space $\widetilde{B}_{a . p .}^{\varphi}(\mathbb{R})$ is strictly convex if and only if $\varphi(\cdot, \cdot)$ is strictly convex and satisfies the $\Delta_{2}$-condition.

Ergodicity in Stepanov-Orlicz spaces was investigated in [153]. Let us recall that a convex function $\phi: \mathbb{R} \longrightarrow[0, \infty)$ is said to be an Orlicz function if and only if it is nondecreasing, even, and continuous on $\mathbb{R}$ and satisfies $\phi(0)=0$, $\phi(u)>0$ for $u>0$, and $\lim _{u \longrightarrow+\infty} \phi(u)=+\infty$. In the newly arisen situation, we say that the function $\phi(\cdot)$ satisfies the $\Delta_{2}$-condition if and only if there exist real numbers $k>1$ and $u_{0}>0$ such that $\phi(2 u) \leq k \varphi(u)$ for $|u| \geq u_{0}$. For any Orlicz function $\phi: \mathbb{R} \longrightarrow[0, \infty)$, it can be simply proved that $f \in \operatorname{PAP}_{0}(\mathbb{R}: X)$ if and only if $\phi(\|f\|) \in \operatorname{PAP}_{0}(\mathbb{R}: X)$. Here, $\mathrm{PAP}_{0}(\mathbb{R}: X)$ stands for the space consisting of all pseudoergodic components, i.e., the bounded continuous functions $\Phi: \mathbb{R} \longrightarrow X$, such that

$$
\lim _{l \rightarrow \infty} \frac{1}{2 l} \int_{-l}^{l}\|\Phi(s)\| \mathrm{d} s=0 .
$$

For any vector-valued measurable function $f: \mathbb{R} \longrightarrow X$, we define the positive function

$$
\rho_{S^{\phi}}(f):=\sup _{x \in \mathbb{R}} \int_{x}^{x+1} \phi(\|f(s)\|) \mathrm{d} s .
$$

The Stepanov-Orlicz function space generated by $\phi$ is defined by

$$
B S^{\phi}(\mathbb{R}, X):=\left\{f \in M(\mathbb{R}: X) ;(\exists \alpha>0) \rho_{S^{\phi}}(\alpha f)<\infty\right\} .
$$

We know that the vector space $B S^{\phi}(\mathbb{R}, X)$ equipped with the Luxemburg norm

$$
\|f\|_{S^{\phi}}:=\inf \left\{k>0: \sup _{x \in \mathbb{R}} \int_{x}^{x+1} \phi(\|f(s)\| / k) \mathrm{d} s \leq 1\right\},
$$

is a Banach space. It is also worth noting that the MorseTransue space type

$$
\widetilde{B S}^{\phi}(\mathbb{R}, X):=\left\{f \in M(\mathbb{R}, X) ;(\exists \alpha>0) \rho_{S^{\phi}}(\alpha f)<\infty\right\},
$$

equipped with the Luxemburg norm, is a closed subspace of $B S^{\phi}(\mathbb{R}, X)$, which is commonly called the Besicovitch-Orlicz class. We know that $B S^{\phi}(\mathbb{R}, X)=\widetilde{B S}^{\phi}(\mathbb{R}, X)$ if and only if $\phi(\cdot)$ satisfies the $\Delta_{2}$-condition.

Furthermore, for any $p \in C_{+}(\mathbb{R})$, we define the Musielak-Orlicz modular-type space

$$
\begin{aligned}
B S^{p(\cdot)}(\mathbb{R}, X):= & \left\{f \in M(\mathbb{R}: X) ;(\exists \alpha>0) \sup _{x \in \mathbb{R}}\right. \\
& \left.\cdot \int_{x}^{x+1}(\|f(s)\| / k)^{p(s)} \mathrm{d} s \leq 1\right\} .
\end{aligned}
$$

For any function $f \in B S^{p(\cdot)}(\mathbb{R}, X)$, the notion of $B S^{p(\cdot)}(\mathbb{R}, X)$-ergodicity in the norm sense and the notion of $B S^{p(\cdot)}(\mathbb{R}, X)$-ergodicity in the modular sense are introduced in [153], Definition 3.1, and [153], Definition 3.2, respectively. Due to [153], Proposition 3.4, these concepts are equivalent.

Let $\phi: \mathbb{R} \longrightarrow[0, \infty)$ be an Orlicz function. In [153], Definition 3.6, the authors introduced the notions of norm ergodicity in Stepanov-Orlicz sense, modular ergodicity in Stepanov-Orlicz sense, and strongly modular ergodicity in Stepanov-Orlicz sense for a given function $f \in B S^{\phi}(\mathbb{R}, X)$. After that, the authors further explored these notions in [153], Theorems 3.8, 3.10, and 3.11, and provided several illustrative examples in [153], Section 4.

Density theorems for almost periodic functions in Hilbert spaces: in this section, we will inscribe a few relevant results obtained by Haraux and Komornik in [154]; these results have been obtained in their investigation of the oscillatory properties of the wave equation. Denote $X_{T}$ the vector space of all square-integrable functions with zero mean by $X_{T}$ :

$$
X_{T}:=\left\{f \in L_{\mathrm{loc}}^{2}(\mathbb{R}: \mathbb{C}) ; f(t+T) \equiv f(t), \int_{0}^{T} f(t) \mathrm{d} t=0\right\},
$$

where $T>0$. If the set $A=\left\{T_{1}, \ldots, T_{N}\right\}$ is a given set of positive real numbers, we define $X:=X_{T_{1}}+\cdots+X_{T_{N}}$.

If $V$ is a certain collection of complex-valued functions and $I$ is an interval in $\mathbb{R}$, then we set $V_{I}:=\left\{f_{I}: f \in V\right\}$. In [154], Theorem 1, the authors proved that there exists a positive real number $T(A)$ such that, for any interval $I \subseteq \mathbb{R}$, we have

$$
X_{I} \text { is dense in } L^{2}(I) \text { if and only if }|I|<T(A),
$$

where $|I|$ denotes the length of interval $I$; furthermore, the orthogonal complement of $X_{I}$ in $L^{2}(I)$ is finite-dimensional 
if $|I|=T(A)$ and infinite-dimensional if $|I|>T(A)$. Suppose that $|I|=T(A)$ and the orthogonal complement of $X_{I}$ in $L^{2}(I)$ is $p$-dimensional for some integer $p \in \mathbb{N}$. If $P_{p-1}$ denotes the vector space consisting of all complex polynomials of degree $\leq p-1$ (also including the zero polynomial), then in [154], Theorem 3(a), it is stated that $Y_{I}$ is dense in $L^{2}(I)$, where $Y:=P_{p-1}+X$; furthermore, $Y_{I}=L^{2}(I)$ if and only if $p=1$, which is equivalent to saying that $\left(P_{i} / P_{j}\right) \in \mathbb{Q}$ for $1 \leq i \leq j \leq N$. Due to [154], Theorem 3(b), there exists a real-valued function $h \in L^{2}(I)$ such that the functions $h, h^{\prime}, \cdots, h^{p-1}$ span $X_{I}$; furthermore, if we extend the function $h(\cdot)$ by zero to the whole real line and denote the obtained function by $H(\cdot)$, then we know that the function $H(\cdot)$ is a nonzero finite linear combination of Dirac measures.

Almost periodicity in chaos: in this section, we will only draw the attention of the readers to the results presented in the tenth section of the recent research monograph [155] by Akhmet. In [155], Section 10, the author investigated the dynamical properties of the following system:

$$
y^{\prime}=A y+G(t, y)+H(x(t)), \quad t \in \mathbb{R},
$$

where $G: \mathbb{R} \times \mathbb{R}^{n} \longrightarrow \mathbb{R}^{n}$ is continuous in both variables and almost periodic in variable $t$ uniformly for $y \in \mathbb{R}^{n}$, the function $H: \mathbb{R}^{m} \longrightarrow \mathbb{R}^{n}$ is continuous, and all eigenvalues of the constant $n \times n$ real matrix $A$ have negative real parts. Roughly speaking, if the perturbation part $H(x(t))$ is chaotic in a certain sense, then system (41) has the interesting feature of chaos with infinitely many almost periodic motions. The obtained results are well illustrated with several numerical tests involving the coupled Duffing oscillators, for which it is well known that they play an important role in modeling of the enhanced signal propagation. The most important notion used in [155], Section 10, is the notion of the Li-Yorke chaotic set with infinitely many almost periodic motions, which is introduced in [155], Definition 10.1, for the equicontinuous families of uniformly bounded functions $x: \mathbb{R} \longrightarrow \Lambda$, where $\Lambda$ is a nonempty compact subset of $\mathbb{R}^{m}$. We would like to note here that this notion can be introduced in the infinite-dimensional setting, even for other types of chaos such as distributional chaos or mean Li-Yorke chaos [156].

Almost periodicity in mathematical biology: there exist numerous research articles concerning almost periodic- and almost automorphic-type solutions for various classes of ordinary and partial differential equations appearing in mathematical biology (see, e.g., the recent article [157] by Abbas, Dama, Pinto, and Sepulveda, monograph [5], and the references quoted therein). In this section, we will present the main details of the investigation [158] carried out by Ding, Liang, and Xiao and the investigation [159] carried out by Zhang, Yang, and Wang. The nonlinear functional differential equation

$$
x^{\prime}(t)=-a x(t)+\frac{p}{1+x^{n}(t-\tau)}, \quad n>0,
$$

was proposed by Mackey and Glass [160] for modeling of hematopoiesis describing the process of production of all types of blood cells generated by a remarkable self-regulated system that is responsive to the demands put upon it. The authors of [158] studied the following modification of (42):

$$
x^{\prime}(t)=-a(t) x(t)+\frac{p(t) x^{l}(t-\tau(t))}{1+x^{l}(t-\tau(t))}, \quad n>0,
$$

where $a, p, \tau: \mathbb{R} \longrightarrow(0, \infty)$ are almost periodic functions, $0<m \leq 1$, and $l>0$. The authors of [158] employed a fixedpoint theorem in cones to achieve their aims. The authors of [159] considered the existence and global exponential convergence of positive almost periodic solutions for the generalized model of hematopoiesis, described by the following nonlinear functional differential equation:

$$
x^{\prime}(t)=-a(t) x(t)+\sum_{i=1}^{m} \frac{b_{i}(t)}{1+x^{n}\left(t-\tau_{i}(t)\right)}, \quad n>0,
$$

where $a, b_{i}, \tau_{i}: \mathbb{R} \longrightarrow(0, \infty)$ are continuous functions for $i=1,2, \ldots, m$; clearly, this equation is a generalization of (42). This model has been proposed by Gyori and Ladas to describe the dynamics of hematopoiesis, i.e., blood cell production. In any reasonable biological interpretation of model (44), only positive functions $x(\cdot)$ can be accepted as solutions. The main results of [159] are Theorems 3.1 and 3.2, in which the authors assumed that $a, b_{i}, \tau_{i}: \mathbb{R} \longrightarrow(0, \infty)$ are almost periodic functions for $i=1,2, \ldots, m$. Set

$$
\begin{aligned}
a^{-} & =\inf _{t \in \mathbb{R}} a(t), a^{+}=\sup _{t \in \mathbb{R}} a(t), b_{i}^{-}=\inf _{t \in \mathbb{R}} b_{i}(t)>0, b_{i}^{+} \\
& =\sup _{t \in \mathbb{R}} b_{i}(t), \\
r & =\max _{1 \leq q \leq n} \sup _{t \in \mathbb{R}} \tau_{i}(t)>0, M_{1}:=\frac{\sum_{i=1}^{m} b_{i}^{+}}{a^{-}}, M_{2} \\
& :=\frac{\sum_{i=1}^{m} b_{i}^{-}}{a^{+}\left(1+M_{1}^{n}\right)},
\end{aligned}
$$

and suppose that $n \sum_{i=1}^{m} b_{i}^{+}<a^{-}$.

Then, there exists a unique positive almost periodic solution of (44) in the closed set $B^{*}=\{f \in$ $\left.A P(\mathbb{R}: \mathbb{R}) ; M_{2} \leq\|f\|_{\infty} \leq M_{1}\right\}$. If we denotex* $(\cdot)$ this solution by $x^{*}(\cdot)$, then any solution $x\left(t ; t_{0}, \varphi\right)$ of equation (44) equipped with the initial condition

$$
x_{t_{0}}=\varphi, \quad \varphi \in C_{+}, \varphi(0)>0
$$

converges exponentially to $x^{*}(t)$ as $t \longrightarrow+\infty$; see [159] for the notion and more details.

Interpolation by periodic and almost periodic functions: the problems of interpolation by periodic and almost periodic functions were intensively studied by a group of Polish mathematicians during the 1960s. Probably, the first fundamental result in this direction was obtained in 1961 by Mycielski [161], who proved that there exists a sequence $\left(t_{n}\right)$ of positive real numbers such that, for every sequence $\left(\varepsilon_{n}\right)$ in $\{0,1\}$, there exists a continuous periodic function $f: \mathbb{R} \longrightarrow \mathbb{C}$ such that $f\left(t_{n}\right)=\varepsilon_{n}$ for all $n \in \mathbb{N}$, answering a question proposed earlier by Marczewski and Ryll- 
Nardzewski. Two years later, this result was extended by Lipiński in [162], who proved that there exists a sequence $\left(t_{n}\right)$ of positive real numbers such that, for every bounded real function $g(\cdot)$ defined on the set $\left\{t_{n}: n \in \mathbb{N}\right\}$, there exists a continuous periodic function $f: \mathbb{R} \longrightarrow \mathbb{C}$ such that $f\left(t_{n}\right)=g\left(t_{n}\right)$ for all $n \in \mathbb{N}$. The essential thing in the aforementioned results is a rapid increase of the sequence $\left(t_{n}\right)$ as $n \longrightarrow+\infty$ : in [161], we concretely have that $t_{n}=(3+\alpha)^{n}$, where $\alpha>0$. Let us note that Ryll-Nardzewski showed that, for every sequence $\left(\varepsilon_{n}\right)$ in $\{0,1\}$, there exists a continuous periodic function $f: \mathbb{R} \longrightarrow \mathbb{C}$ such that $f\left(3^{n}\right)=$ $\varepsilon_{n}$ for all $n \in \mathbb{N}$ as well as that there does not exist a sequence $\left(t_{n}\right)$ of positive real numbers with $t_{n}=O\left(2^{n}\right), n \in \mathbb{N}$, satisfying the above property. Interpolation by almost periodic functions was investigated for the first time by Hartman [163] in 1961 and later reconsidered in a series of his joint research papers with Ryll-Nardzewski [164-166] during the period 1964-1967. In [164], the authors analyzed the following properties for the subset $\Lambda$ of the real line $\mathbb{R}$ (and the abelian topological groups):

$I: \Lambda$ satisfies property $I$ if and only if any bounded, uniformly continuous function $g: \Lambda \longrightarrow \mathbb{C}$ can be extended to an almost periodic function $f: \mathbb{R} \longrightarrow \mathbb{C}$

$I_{0}: \Lambda$ satisfies property $I_{0}$ if and only if any bounded function $g: \Lambda \longrightarrow \mathbb{C}$ can be extended to an almost periodic function $f: \mathbb{R} \longrightarrow \mathbb{C}$

The authors first proved that there are no sequence $\left(\varepsilon_{n}\right)$ in $\{0,1\}$ and an almost periodic function $f: \mathbb{R} \longrightarrow \mathbb{C}$ such that $f\left(n^{\alpha}\right)=\varepsilon_{n}$ for all $n \in \mathbb{N}$, provided that $\alpha>0$ is not an integer; this essentially follows from the equality

$$
\lim _{N \longrightarrow \infty} \frac{1}{N} \sum_{n=1}^{N} f\left(n^{\alpha}\right)=\lim _{T \longrightarrow \infty} \frac{1}{2 T} \int_{-T}^{T} f(t) \mathrm{d} t,
$$

which is valid for these values of number $\alpha>0$. The main results concerning properties $I$ and $I_{0}$ and extensions to uniformly continuous almost periodic functions were proved in [164], Theorems 1 and 2, while the third main result of this paper, [164], Theorem 3, analyzes a similar problem for extensions to Stepanov almost periodic functions. In [167], Strzelecki proved that any sequence $\left(t_{n}\right)$ of positive real numbers such that $\left(t_{n+1} / t_{n}\right)>1+\delta, n \in \mathbb{N}$, where $\delta>0$ is a fixed real number, has property $I_{0}$; later, this result was extended in [165], Theorem 5. Interpolation by Levitan almost periodic functions was considered by Hartman in [168].

In the list of [5], we have also quoted some references concerning subjects such as the Bohr compactifications, almost periodic functions on $C^{*}$-algebras, semiholomorphic almost periodic functions, and certain interplays between the almost periodicity and the representation theory.

\section{Almost Periodic Functions of Several Real Variables and Their Applications}

The notion of almost periodicity can be simply generalized to the case in which $I=\mathbb{R}^{n}$. Suppose that $F: \mathbb{R}^{n} \longrightarrow X$ is a continuous function. Then, we say that $F(\cdot)$ is almost periodic if and only if for each $\varepsilon>0$, there exists $l>0$ such that, for each $\mathbf{t}_{0} \in \mathbb{R}^{n}$, there exists $\tau \in B\left(\mathbf{t}_{0}, l\right)$ such that

$$
\|F(\mathbf{t}+\tau)-F(\mathbf{t})\| \leq \varepsilon, \quad \mathbf{t} \in \mathbb{R}^{n} .
$$

This is equivalent to saying that, for any sequence $\left(\mathbf{b}_{n}\right)$ in $\mathbb{R}^{n}$, there exists a subsequence $\left(\mathbf{a}_{n}\right)$ of $\left(\mathbf{b}_{n}\right)$ such that $(F(\cdot+$ $\left.\mathbf{a}_{n}\right)$ ) converges in $C_{b}\left(\mathbb{R}^{n}: X\right)$. Any trigonometric polynomial in $\mathbb{R}^{n}$ is almost periodic, and it is also well known that $F(\cdot)$ is almost periodic if and only if there exists a sequence of trigonometric polynomials in $\mathbb{R}^{n}$ which converges uniformly to $F(\cdot)$; let us recall that a trigonometric polynomial in $\mathbb{R}^{n}$ is any linear combination of functions such as $\mathbf{t} \longmapsto e^{i\langle\lambda, \mathbf{t}\rangle}$, $\mathbf{t} \in \mathbb{R}^{n}$, where $\lambda \in \mathbb{R}^{n}$ and $\langle\cdot, \cdot\rangle$ denotes the inner product in $\mathbb{R}^{n}$. Any almost periodic function $F: \mathbb{R}^{n} \longrightarrow X$ is almost periodic with respect to each of the variables, but the converse statement is not true since the function $\left(t_{1}, t_{2}\right) \longmapsto \cos \left(t_{1} t_{2}\right), t_{1}, t_{2} \in \mathbb{R}$, is almost periodic with respect to both variables $t_{1}$ and $t_{2}$ but not almost periodic with respect to $\left(t_{1}, t_{2}\right)$. Furthermore, for any almost periodic function $F(\cdot)$, we have that, for each $\varepsilon>0$, there exists $l>0$ such that, for each $\mathbf{t}_{0} \in\{(t, t, \ldots, t): t \in \mathbb{R}\}$, there exists $\tau \in B\left(\mathbf{t}_{0}, l\right) \cap\{(t, t, \ldots, t): t \in \mathbb{R}\}$ such that (48) holds. Any almost periodic function $F(\cdot)$ is bounded, the mean value

$$
M(F):=\lim _{T \longrightarrow+\infty} \frac{1}{(2 T)^{n}} \int_{s+K_{T}} F(\mathbf{t}) \mathrm{d} \mathbf{t},
$$

exists, and it does not depend on $s \in \mathbb{R}^{n}$; here, $K_{T}:=\left\{\mathbf{t}=\left(t_{1}, t_{2}, \ldots, t_{n}\right) \in \mathbb{R}^{n}:\left|t_{i}\right| \leq T\right.$, for $\left.1 \leq i \leq n\right\}$. The Bohr-Fourier coefficient $F_{\lambda} \in X$ is defined by

$$
F_{\lambda}:=M\left(e^{-i\langle\lambda, \cdot\rangle} F(\cdot)\right), \quad \lambda \in \mathbb{R}^{n},
$$

where $\langle\cdot, \cdot\rangle$ denotes the usual inner product in $\mathbb{R}^{n}$. The Bohr spectrum of $F(\cdot)$, defined by $\sigma(F):=\left\{\lambda \in \mathbb{R}^{n}: F_{\lambda} \neq 0\right\}$, is at most a countable set.

The almost periodic functions of two real variables are also investigated by Besicovitch in the classic [169]. Here, we would like to note that the results established in [169] can be straightforwardly generalized to the almost periodic functions of several real variables. For example, if $t_{i}$ is a fixed variable from the set $\left\{t_{1}, \ldots, t_{n}\right\}$, then the function $t_{i} \longmapsto$ $F\left(t_{1}, \ldots, t_{i}, \ldots, t_{n}\right), t_{i} \in \mathbb{R}$, is almost periodic for every fixed real number $t_{1}, \ldots, t_{i-1}, t_{i+1}, \ldots, t_{n}$ so that the mean value

$$
\begin{aligned}
M_{t_{i}}\left\{F\left(t_{1}, \ldots, t_{n}\right)\right\}:= & \lim _{T_{i}} \frac{1}{\longrightarrow+\infty} \frac{1}{2 T_{i}} \\
& \cdot \int_{-T_{i}}^{T_{i}} F\left(t_{1}, \ldots, t_{i}, \ldots, t_{n}\right) \mathrm{d} t_{i},
\end{aligned}
$$

exists. Considering $M_{t_{i}}\left\{F\left(t_{1}, \ldots, t_{n}\right)\right\}$ as a function of the variables $t_{1}, \ldots, t_{i-1}, t_{i+1}, \ldots, t_{n}$, it can be easily shown that it is almost periodic in $\mathbb{R}^{n-1}$. Therefore, we can calculate the repeated mean value

$$
\begin{aligned}
& \left(M_{t_{j}} \circ M_{t_{i}}\right)\left\{F\left(t_{1}, \ldots, t_{n}\right)\right\} \\
& \quad:=\lim _{T_{j}} \underset{\longrightarrow+\infty}{ } \frac{1}{2 T_{j}} \int_{-T_{j}}^{T_{j}} M_{t_{i}}\left\{F\left(t_{1}, \ldots, t_{n}\right)\right\} \mathrm{d} t_{j},
\end{aligned}
$$


for any fixed real numbers from the set $\left\{t_{1}, \ldots, t_{n}\right\} \backslash\left\{t_{i}, t_{j}\right\}$. If we fix these numbers in advance, we can apply ([169], Corollary, p. 63) to the almost periodic function

$$
F_{i j}\left(t_{i}, t_{j}\right):=F\left(t_{1}, \ldots, t_{i}, \ldots, t_{j}, \ldots, t_{n}\right), \quad\left(t_{i}, t_{j}\right) \in \mathbb{R}^{2},
$$

in order to see that

$$
\left(M_{t_{j}} \circ M_{t_{i}}\right)\left\{F\left(t_{1}, \ldots, t_{n}\right)\right\} \equiv\left(M_{t_{i}} \circ M_{t_{j}}\right)\left\{F\left(t_{1}, \ldots, t_{n}\right)\right\}
$$

Inductively, we easily get that, for every finite tuple of different variables $\left(t_{i_{1}}, \ldots, t_{i_{l}}\right)$, where $1 \leq i_{1}<i_{2}<\cdots<i_{l} \leq n$, and for every permutation $\sigma:\left\{i_{1}, \ldots, i_{l}\right\} \longrightarrow\left\{i_{1}, \ldots, i_{l}\right\}$, we have

$$
\begin{aligned}
& \left(M_{t_{i_{1}}} \circ \ldots \circ M_{t_{i_{l}}}\right)\left\{F\left(t_{1}, \ldots, t_{n}\right)\right\} \\
& \quad=\left(M_{t_{\sigma\left(i_{1}\right)}} \circ \cdots \circ M_{t_{\sigma\left(i_{l}\right)}}\right)\left\{F\left(t_{1}, \ldots, t_{n}\right)\right\} .
\end{aligned}
$$

By $A P\left(\mathbb{R}^{n}: X\right)$ and $A P_{\Lambda}\left(\mathbb{R}^{n}: X\right)$, we denote, respectively, the Banach space consisting of all almost periodic functions $F: \mathbb{R}^{n} \longrightarrow X$, equipped with the sup-norm, and its subspace consisting of all almost periodic functions $F: \mathbb{R}^{n} \longrightarrow X$ such that $\sigma(F) \subseteq \Lambda$. As is well known, for every almost periodic function $F \in A P_{\Lambda}\left(\mathbb{R}^{n}: X\right)$, we can always find a sequence $\left(P_{k}\right)$ of trigonometric polynomials in $\mathbb{R}^{n}$ which uniformly converges to $F(\cdot)$ on $\mathbb{R}^{n}$ and satisfies that $\sigma\left(P_{k}\right) \subseteq \Lambda$ for all $k \in \mathbb{N}$; see, e.g., [170], Chapter 1, Section 2.3. The Wiener algebra APW $\left(\mathbb{R}^{n}: X\right)$ is defined as the set of all functions $F: \mathbb{R}^{n} \longrightarrow X$ such that its Fourier series converges absolutely; $\mathrm{APW}_{\Lambda}\left(\mathbb{R}^{n}: X\right) \equiv \operatorname{APW}\left(\mathbb{R}^{n}: X\right) \cap A P_{\Lambda}\left(\mathbb{R}^{n}: X\right)$. It is well known that the Wiener algebra is a Banach algebra with respect to the Wiener norm $\|F\|:=\sum_{\lambda \in \mathbb{R}^{n}}\left|F_{\lambda}\right|$, $F \in \operatorname{APW}\left(\mathbb{R}^{n}: X\right)$, as well as that $\operatorname{APW}\left(\mathbb{R}^{n}: X\right)$ is dense in $A P\left(\mathbb{R}^{n}: X\right)$.

The theory of almost periodic functions of several real variables has not attracted so much attention compared with the theory of almost periodic functions of one real variable by now. In the following, we will remind the readers of several important investigations of multidimensional almost periodic functions carried out so far:

1. Problems of Nehari type and contractive extension problems for matrix-valued (Wiener) almost periodic functions of several real variables have been considered by Rodman, Spitkovsky, and Woerdeman in [171], where the authors proved a generalization of the famous Sarason's theorem. In their analysis, the notion of a half-space in $\mathbb{R}^{n}$ plays an important role: a nonempty subset $S \subseteq \mathbb{R}^{n}$ is said to be a half-space if and only if the following four conditions hold:

(i) $\mathbb{R}^{n}=S \cup(-S)$

(ii) $\{0\}=S \cap(-S)$

(iii) $S+S \subseteq S$

(iv) $\alpha \cdot S \subseteq S$ for $\alpha \geq 0$
For any half-space $S$, we can always find a linear bijective mapping $D: \mathbb{R}^{n} \longrightarrow \mathbb{R}^{n}$ such that $S=D E_{n}$, where $E_{n}$ is a very special half-space defined on [172], p. 3190. In [172], Theorem 1.3, Rodman and Spitkovsky proved that if $S$ is a half-space and $\Lambda \subseteq S$, $0 \in \Lambda$, and $\Lambda+\Lambda \subseteq \Lambda$, then $A P_{\Lambda}\left(\mathbb{R}^{n}: \mathbb{C}\right)$ and $\mathrm{APW}_{\Lambda}\left(\mathbb{R}^{n}: \mathbb{C}\right)$ are Hermitian rings. See also [173].

(2) Let us recall that a subset $\Lambda$ of $\mathbb{R}^{n}$ is called discrete if and only if any point $\lambda \in \Lambda$ is isolated in $\Lambda$. By $\mathscr{V}_{\Lambda}$, we denote the vector space of all finite complexvalued trigonometric polynomials $\sum_{\lambda \in \Lambda} c(\lambda) e^{-\pi i \lambda}$. whose frequencies $\lambda$ belong to $\Lambda$. The space of meanperiodic functions with the spectrum $\Lambda$, denoted by $\mathscr{C}_{\Lambda}$, is defined as the closure of the space $\mathscr{V}_{\Lambda}$ in the Fréchet space $C\left(\mathbb{R}^{n}\right)$. Clearly, $A P_{\Lambda}\left(\mathbb{R}^{n}: \mathbb{C}\right)$ is contained in $\mathscr{C}_{\Lambda}$, but the converse statement is not true, in general. The problem of describing the structure of closed discrete sets $\Lambda$ for which the equality $A P_{\Lambda}\left(\mathbb{R}^{n}: \mathbb{C}\right)=\mathscr{C}_{\Lambda}$ holds was proposed by Kahane in 1957. For more details about this interesting problem, we refer the reader to the survey article [174] by Meyer; for more details about mean-periodic functions, see also the lectures by Kahane [175].

(3) In 1971, Basit [176] observed that there exists a complex-valued almost periodic function $f: \mathbb{R}^{2} \longrightarrow \mathbb{C}$ such that the function $F: \mathbb{R}^{2} \longrightarrow \mathbb{C}$, defined by $F(x, y):=\int_{0}^{x} f(t, y) \mathrm{d} t,(x, y) \in \mathbb{R}^{2}$, is bounded but not almost periodic. This result was recently reconsidered by Alsulami in [177], Theorem 2.2, who proved that, for a complex-valued almost periodic function $f: \mathbb{R}^{2} \longrightarrow \mathbb{C}$, the boundedness of the function $F(\cdot)$ in the whole plane implies its almost periodicity, provided that there exists a complex-valued almost periodic function $g: \mathbb{R}^{2} \longrightarrow \mathbb{C}$ such that $f_{x}(x, y)=g_{y}(x, y)$ is a continuous function in the whole plane. This result was proved with the help of an old result of Loomis which states that, for a bounded complex-valued function $f: \mathbb{R}^{n} \longrightarrow \mathbb{C}$, the almost periodicity of all its partial derivatives of the first order implies the almost periodicity of $f(\cdot)$ itself. Let us observe that the aforementioned result of Alsulami can be straightforwardly extended, with the same proof, to the almost periodic functions $f: \mathbb{R}^{n} \longrightarrow \mathbb{C}$; in actual fact, if the function $f: \mathbb{R}^{n} \longrightarrow \mathbb{C}$ is almost periodic, the function $F\left(x_{1}, x_{2}, \ldots, x_{n}\right):=$ $\int_{0}^{x_{1}} f\left(t, x_{2}, \ldots, x_{n}\right) \mathrm{d} t, \quad\left(x_{1}, x_{2}, \ldots, x_{n}\right) \in \mathbb{R}^{n}$ is bounded, and there exist almost periodic functions $\quad G_{i}: \mathbb{R}^{n} \longrightarrow \mathbb{C}$ such that $F_{x_{i}}\left(x_{1}, x_{2}, \ldots, x_{n}\right)=\left(G_{i}\right)_{x_{1}}\left(x_{1}, x_{2}, \ldots, x_{n}\right)$ is a continuous function on $\mathbb{R}^{n}$, for $2 \leq i \leq n$, then the function $F: \mathbb{R}^{n} \longrightarrow \mathbb{C}$ is almost periodic.

(4) In [178-183], Khasanov investigated the approximations of uniformly almost periodic functions of two variables by partial sums of Fourier sums and Marcinkiewicz sums in the uniform metric, provided certain conditions. 
(5) In $[184,185]$, Latif and Bhatti investigated several important questions concerning almost periodic functions defined on $\mathbb{R}^{n}$ with values in locally convex spaces and fuzzy-number-type spaces (almost periodic functions defined on $\mathbb{R}^{n}$ with values in $p$-Fréchet spaces, where $0<p<1$, were investigated in [186] by N'Guérékata, Latif, and Bhatti).

Concerning applications made so far, we recall the following:

(1 ) The problem of the existence of almost periodic solutions for the system of linear partial differential equations $\sum_{j=1}^{n} L_{i j} u_{j}=f_{i}, 1 \leq i \leq n$, on $\mathbb{R}^{m}$, where $L_{i j}$ is an arbitrary linear partial differential operator on $\mathbb{R}^{m}$, was analyzed by Sell $[187,188]$. He extended the results obtained by Sibuya, where the author analyzed the almost periodic solutions of Poisson's equation.

(2) The almost periodic solutions of the (semilinear) systems of ordinary differential equations were analyzed by Fink in [189], Chapter 8, with the help of fixed-point theorems. Furthermore, Liu Bao-Ping and Pao investigated the almost periodic plane wave solutions of certain classes of coupled nonlinear reaction-diffusion equations [190]; in their approach, a solution $u(t, x)$ of such a system, where $t \in \mathbb{R}$ and $x \in \mathbb{R}^{n}$, is almost periodic in $\mathbb{R}^{n+1}$ and satisfies that $u(t, x)$ is almost periodic in the time variable $t \in \mathbb{R}$ and periodic in each spatial variable (see [190], Theorem 2).

(3) In his doctoral dissertation [191], Alsulami considered the question whether the boundedness of solutions of the following system of partial first-order differential equations

$$
\begin{aligned}
& u_{s}(s, t)=A u(s, t)+f_{1}(s, t), \\
& u_{t}(s, t)=B u(s, t)+f_{2}(s, t), \quad(s, t) \in \mathbb{R}^{2},
\end{aligned}
$$

implies the almost periodicity of solutions to (56). He analyzed this question in the finite-dimensional setting and the infinite-dimensional setting, using two different techniques; in both cases, $A$ and $B$ are bounded linear operators acting on the pivot space $X$.

(4) In [192-194], Spradlin provided several interesting results and applications regarding almost periodic functions of several real variables. The existence of positive homoclinic-type solutions of the equation

$$
-\Delta u+u=H(\mathbf{t}) f(u),
$$

where $H(\cdot)$ is almost periodic and the first integral of $f(\cdot)$ satisfies certain superquadraticity and critical growth conditions, has been analyzed in [194], Theorem 1.2. The equations of type

$$
-\varepsilon^{2} \Delta u+H(t) u=f(u),
$$

arise in the study of the nonlinear Schrödinger equations $(\varepsilon>0)$. A qualitative analysis of solutions of (58) has been carried out in [193], provided the almost periodicity of function $H(\cdot)$ and several other nontrivial assumptions.

(5) The existence and uniqueness of almost periodic solutions for a class of boundary value problems for hyperbolic equations were investigated by Ptashnic and Shtabalyuk in [195] (also, cf. the sixth chapter in monograph [53] by Ptashnic). In the region $D_{p}=$ $(0, T) \times \mathbb{R}^{p}(T>0, p \in \mathbb{N})$, they have analyzed the well-posedness of the following initial value problem:

$$
\begin{aligned}
L u \equiv & \sum_{s=0|\alpha|=2 s}^{n} \sum_{\alpha} \frac{\partial^{2 n} u(t, x)}{\partial t^{2 n-2 s} \partial x_{1}^{\alpha_{1}} \ldots \partial x_{p}^{\alpha_{p}}}=0, \\
& \left.\frac{\partial^{j-1} u}{\partial t^{j-1}}\right|_{t=0}=\varphi_{j}(x), \\
& \left.\frac{\partial^{j-1} u}{\partial t^{j-1}}\right|_{t=T}=\varphi_{j+n}(x), \quad(1 \leq j \leq n) .
\end{aligned}
$$

The basic assumption employed in [195] is that equation (59) is Petrovsky-hyperbolic, i.e., for each $\mu=\left(\mu_{1}, \mu_{2}, \ldots, \mu_{p}\right) \in \mathbb{R}^{p}$, all $\lambda$-zeroes of the equation

$$
\sum_{s=0|\alpha|=2 s}^{n} a_{\alpha} \lambda^{2 n-2 s} \mu_{1}^{\alpha_{1}} \mu_{2}^{\alpha_{2}} \ldots \mu_{p}^{\alpha_{p}}=0,
$$

are real. The basic function space used is the Banach space $C_{B}^{q}\left(\overline{D^{p}}\right)$ consisting of all $q$-times continuously differentiable functions $u(t, x)$ in $\overline{D^{p}}$ that are Bohr almost periodic in variables $x_{1}, x_{2}, \ldots, x_{p}$, uniformly in $t \in[0, T]$, equipped with the norm

$$
\|u\|_{C_{B}^{q}\left(\overline{D^{p}}\right)}:=\sup _{0 \leq|s| \leq q} \sup _{(t, x) \in \overline{D^{p}}} \frac{\partial^{|s|} u(t, x)}{\partial t^{s_{0}} \partial x_{1}^{s_{1}} \ldots \partial x_{p}^{s_{p}}},
$$

and by $C_{B}^{q}\left(\mathbb{R}^{p}\right)$, the authors designated the subspace of $C_{B}^{q}\left(\overline{D^{p}}\right)$ consisting of those functions which do not depend on variable $t$. The existence and uniqueness of solutions of initial value problems (59) and (60) have been investigated in the space $C_{B}^{2 n}\left(\overline{D^{p}}\right)$, under the assumption that $\varphi_{j}(x) \in C_{B}^{r}\left(\mathbb{R}^{p}\right)$ and $r \in \mathbb{N}$ is sufficiently large. If $M_{p}=\left\{\mu_{k}: k \in \mathbb{Z}^{p}\right\}$ is the union of spectrum of all functions $\varphi_{1}(x), \ldots, \varphi_{2 n}(x)$, the solutions $u(t, x)$ of problems (59) and (60) have been found in the form

$$
u(t, x)=\sum_{k \in \mathbb{Z}^{p}} u_{k}(t) e^{i\left\langle\mu_{k}, x\right\rangle}, \quad \mu_{k} \in M_{p},
$$

where the functions $u_{k}(t)$ satisfy certain conditions and have the form given in equation ([195], (8), p. 670). The uniqueness of solutions of problems (59) and (60) has been considered in [195], Theorem 1, 
while the existence of solutions of problems (59) and (60) has been considered in [195], Theorem 2.

(6) The class of vector-valued remotely almost periodic functions defined on $\mathbb{R}^{n}$ was introduced by Yang and Zhang in [196]. In the same paper, the authors provided several applications in the study of the existence and uniqueness of remotely almost periodic solutions for parabolic boundary value problems. A function $F: \mathbb{R}^{n} \longrightarrow X$ is said to be remotely almost periodic if and only if for each $\varepsilon>0$, the set of all vectors $\tau \in \mathbb{R}^{n}$ for which

$$
\lim \sup _{|\mathbf{t}| \longrightarrow+\infty}\|F(\mathbf{t}+\tau)-F(\mathbf{t})\|<\varepsilon,
$$

is relatively dense in $\mathbb{R}^{n}$ (the vector $\tau$ is called a remotely $\epsilon$-translation vector of $F(\cdot)$ ); furthermore, if $\varnothing \neq \Omega \subseteq \mathbb{R}^{m}$, then a continuous function $F: \mathbb{R}^{n} \times \Omega \longrightarrow X$ is said to be remotely almost periodic in $\mathbf{t} \in \mathbb{R}^{n}$ and uniform on compact subsets of $\Omega$ if and only if $F(\cdot, y)$ is remotely almost periodic for each $y \in \Omega$ and is uniformly continuous on $\mathbb{R}^{n} \times K$ for any compact subset $K \subseteq \Omega$. The following statements hold in the scalar-valued case (see, e.g., [196], Propositions 2.1-2.3):

(i) If $F(\cdot)$, resp. $F(\cdot ; \cdot)$, is remotely almost periodic and the function $\left(\partial F / \partial \mathbf{t}_{i}(\cdot)\right)$, resp. $\left(\partial F / \partial \mathbf{t}_{i}(\cdot ; \cdot)\right)$, is uniformly continuous on $\mathbb{R}^{n}$, then the function $\left(\partial F / \partial \mathbf{t}_{i}\right)$, resp. $\left(\partial F / \partial \mathbf{t}_{i}(\cdot ; \cdot)\right)$, is remotely almost periodic, as well $(1 \leq i \leq n)$.

(ii) If the functions $F_{1}(\cdot), \ldots, F_{k}(\cdot)$ are remotely almost periodic $(k \in \mathbb{N})$, then for each $\varepsilon>0$, the set of their common $\epsilon$-translation vectors is relatively dense in $\mathbb{R}^{n}$.

(iii) If the functions $H_{1}(\cdot), \ldots, H_{k}(\cdot)$ are remotely almost periodic $(k \in \mathbb{N})$ and $\left(H_{1}(t), \ldots, H_{k}(t)\right) \in \Omega$ for all $t \in \mathbb{R}$, then for every remotely almost periodic function $F: \mathbb{R} \times \Omega \longrightarrow \mathbb{C}$, we have that the function

$$
t \longmapsto F\left(H_{1}(t), \ldots, H_{k}(t), t\right), \quad t \in \mathbb{R}
$$

is remotely almost periodic.

In [196], Propositions 2.4-2.6, the authors examined the existence and uniqueness of remotely almost periodic solutions of multidimensional heat equations, while the main results of the third section of this paper are concerned with the existence and uniqueness of remotely almost periodictype solutions of certain types of parabolic boundary value problems.

The boundedness and almost periodicity in time for certain classes of evolution variational inequalities, positive boundary value problems for symmetric hyperbolic systems, and nonlinear Schrödinger equations have been investigated in the third and fourth section of the important research monograph [170] by Pankov (for almost periodic properties of Schrödinger equations and Schrödinger-type operators, see the reference list of [5]). Spatially, Besicovitch almost periodic solutions for certain classes of nonlinear secondorder elliptic equations, first-order hyperbolic systems, single higher-order hyperbolic equations, and nonlinear Schrödinger equations have been investigated in the fifth section of this monograph. For more details about the applications of Stepanov multidimensional almost periodic functions and Weyl multidimensional almost periodic functions, as well as to some interplays between the multidimensional almost periodic functions, calculus of variations, and homogenization theory, we refer the reader to notes and appendices to the third section of [5].

It is worth mentioning that Spradlin constructed, in [192], an almost periodic infinitely differentiable function $G: \mathbb{R}^{n} \longrightarrow \mathbb{R}$ with no local minimum (it can be simply shown that this situation cannot occur in the one-dimensional case because any almost periodic function $g: \mathbb{R} \longrightarrow \mathbb{R}$ must have infinitely many local minima); this important peculiarity of almost periodic functions of several real variables was perceived twenty five years ago. The construction of an almost periodic function $G: \mathbb{R}^{n} \longrightarrow \mathbb{R}$ with no local minimum, established in [192], is very complicated, and the proof of the main result of this paper ([192], Theorem 1.0) contains almost eight pages including some preliminaries. It can be easily proved, by observing that the function $G(x, y)$ is strictly positive, that the function $(x, y) \longmapsto H(x, y) \equiv \int_{0}^{x} G(t, y) \mathrm{d} t$ is bounded and not almost periodic in the plane. As already mentioned, the existence of a complex-valued almost periodic function $H(x, y)$ with these properties was clarified by Basit (1971) with very obscure evidence, not including the smoothness of $G(x, y)$ or its nonnegativity.

At the end of paper [192], Spradlin proposed the following questions:

(1) The almost periodic function $F: \mathbb{R}^{2} \longrightarrow \mathbb{R}$ constructed in the proof of [192], Theorem 1.0, has an absolute maximum at the point $(0,0)$. Does there exist an almost periodic function $F: \mathbb{R}^{n} \longrightarrow \mathbb{R}$ with no local minimum or maximum?

(2) Does there exist a real analytic almost periodic function $F: \mathbb{R}^{n} \longrightarrow \mathbb{R}$ with no local minimum or maximum?

(3) Is it true that a continuously differentiable almost periodic function $F: \mathbb{R}^{n} \longrightarrow \mathbb{R}$ has a critical point?

(4) Does there exist a quasi-periodic function $F: \mathbb{R}^{n} \longrightarrow \mathbb{R}$ with no local minimum (local minimum or maximum)?

To the best of authors' knowledge, all these questions are still open. Concerning open problems, we also want to remind our readers of article [197] by Basit.

Now, we would like to say something more about the following intriguing topics.

Multivariate trigonometric polynomials and approximations of periodic functions of several real variables: without any doubt, trigonometric polynomials of several real variables, sometimes also called multivariate trigonometric polynomials, present the best-explored class of almost periodic functions of several real variables. Multivariate trigonometric polynomials have an invaluable importance in the theory of approximations of periodic functions of several 
real variables, especially in the two-dimensional case. For the basic source of information about this subject, the reader may consult research monographs [198] by Dumitrescu, [199] by Dung, Temlyakov, and Ullrich, and [200, 201] by Temlyakov, research article [202] by Temlyakov, and the list of references quoted in [5].

In this part, we will briefly explain the main results and ideas of papers [203] by Babayev, [204] by Pfister and Bresler, and [205] by Kämmerer, Potts, and Volkmer. If $f: \mathbb{R} \longrightarrow \mathbb{R}$ belongs to the space $C_{2 \pi}$ of all real continuous functions of period $2 \pi$, then it is well known that the Vallee Poussin singular integral $V_{k}(\cdot)$, defined by

$$
\begin{array}{r}
V_{k}(x):=\frac{1}{2 \pi} \frac{(2 k) ! !}{(2 k-1) ! !} \int_{-\pi}^{\pi} f(t) \cos \frac{2 k}{2} \mathrm{~d} t, \\
x \in \mathbb{R}(k \in \mathbb{N}),
\end{array}
$$

has the property that $\lim _{k \longrightarrow+\infty} V_{k}(x)=f(x)$, uniformly for $x \in \mathbb{R}$. This result of Vallee Poussin improves the classical Weierstrass second theorem on the density of trigonometric polynomials in the spaces of continuous functions. Twodimensional Vallee Poussin singular integral $V_{k, m}(\cdot)$, defined for each $x \in \mathbb{R}$ by $(k, m \in \mathbb{N})$,

$$
\begin{aligned}
V_{k, m}(x, y):= & \frac{1}{(2 \pi)^{2}} \frac{(2 k) ! !}{(2 k-1) ! !} \frac{(2 m) ! !}{(2 m-1) ! !} \\
& \cdot \int_{-\pi}^{\pi} f(t, \tau) \cos ^{2 k} \frac{t-x}{2} \cos ^{2 k} \frac{\tau-y}{2} \mathrm{~d} \tau,
\end{aligned}
$$

has been introduced in [203], Definition 2. In the same paper, the author showed that $\lim _{k \longrightarrow+\infty}$ $\lim _{m \longrightarrow+\infty} V_{k, m}(x, y)=f(x, y)$, uniformly for $(x, y) \in \mathbb{R}^{2}$, as well as that $V_{k, m}(x, y)$ is a trigonometric polynomial in variables $x$ and $y$, for all $k, m \in \mathbb{N}$ (see [203], Theorem 2). For proving the last fact, the author used a lemma clarifying that the product of two trigonometric polynomials of two variables is also the trigonometric polynomial of two variables whose order equals the sum of order of cofactors as well as that any even trigonometric polynomial $T(x, y)$, i.e., a trigonometric polynomial $T(x, y)$ which satisfies that $T(-x,-y)=T(x, y), T(-x, y)=T(x, y)$, and $T(x,-y)=$ $T(x ; y)$ identically for $(x, y) \in \mathbb{R}^{2}$, may be represented in the form

$$
\begin{array}{r}
T(x, y)=A+\sum_{k=1}^{m} \sum_{l=1}^{n}\left(a_{k l} \cos k x \cos l y+b_{k l} \cos k x+c_{k l} \cos l y\right), \\
(x, y) \in \mathbb{R}^{2},
\end{array}
$$

which does not contain the sines of multiple arcs (see [203], Lemmas 3 and 4). We would like to note that the obtained results continue to hold in the vector-valued case.

In [204], Pfister and Bresler investigated bounding multivariate trigonometric polynomials and gave some applications to the problems of filter bank design. Denote

$$
\begin{aligned}
T_{l}^{n}:=\operatorname{span}\left\{e^{i\langle\mathbf{k}, \lambda\rangle}: \lambda \in[0,2 \pi]^{n}, \mathbf{k} \in \mathbb{Z}^{n},\|\mathbf{k}\|:=\sup _{1 \leq i \leq n}\left|k_{i}\right| \leq l\right\}, \quad(l \in \mathbb{N}), \\
\Theta_{N}:=\left\{\frac{2 \pi k}{N}: k=0,1, \ldots, N-1\right\}, \quad(N \in \mathbb{N}) .
\end{aligned}
$$

For any $N \in \mathbb{N}$ and for any real-valued trigonometric polynomial

$$
P(\lambda):=\sum_{k_{1}=-l}^{l} \sum_{k_{2}=-l}^{l} \ldots \sum_{k_{n}=-l}^{l} c_{k_{1} k_{2} \ldots k_{n}} e^{i\langle k, \lambda\rangle} \in T_{l}^{n},
$$

i.e., the multivariate trigonometric polynomial $P(\cdot)$ for which $c_{k_{1}, k_{2}, \ldots, k_{n}}=c_{-k_{1},-k_{2}, \ldots,-k_{n}}^{*}(\|\mathbf{k}\| \leq l$; star denotes complex conjugation), we define

$$
\|P\|_{\infty}:=\max _{\lambda \in[0,2 \pi]^{n}}|P(\lambda)| \text { and }\|P\|_{N^{n}, \infty}:=\max _{\lambda \in \Theta_{N}^{n}}|P(\lambda)| \text {. }
$$

Then, two well-known results of the approximation theory state that

$$
\|P\|_{\infty} \leq\|P\|_{(2 l+1)^{n}, \infty}\left(1+4 \pi^{-1}+2 \pi^{-1} \ln (2 l+1)\right)^{n},
$$

and in the one-dimensional case,

$$
\|P\|_{\infty} \leq \frac{\|P\|_{N, \infty}}{\sqrt{1-((2 l / N))}} .
$$

In [204], Theorem 1, the authors showed that the assumptions $N \geq 2 l+1$ and $\alpha=(2 l / N)$ yield the existence of a positive real constant $C_{N, l}^{n} \in\left[0,(1-\alpha)^{-(n / 2)}\right]$ such that

$$
\|P\|_{\infty} \leq C_{N, l}^{n}\|P\|_{N^{n}, \infty}, \quad P \in T_{l}^{n},
$$

and $C_{N, l}^{n}\|P\|_{N^{n}, \infty}-\|P\|_{\infty}=O(\ln / N), P \in T_{l}^{n}$. In order to achieve their aims, the authors used the de la Vallee Poussin kernels and the tensor products of one-dimensional Dirichlet kernels.

In [205], the authors investigated certain algorithms for the approximation of multivariate periodic functions by trigonometric polynomials, which are based on the use of a single one-dimensional fast Fourier transform and the socalled method of sampling of multivariate functions on rank-1 lattices. In their analysis, the authors used periodic Sobolev spaces of generalized mixed smoothness and 
presented some advantages of their method compared to the method based on the trigonometric interpolations on generalized sparse grids. Some numerical results and tests are presented up to dimension $n=10$, as well.

Almost periodic pseudo-differential operators and Gevrey classes: almost periodic pseudo-differential operators have been analyzed by numerous mathematicians including Coburn, Moyer, and Singer [206], Dedik [207], Iannacci, Bersani, Dell'Acqua, and Santucci [208], Pankov [209], Shubin [210-213], and Wahlberg [214]. In this part, we will present the main ideas and results of research study [215] by Oliaro, Rodino, and Wahlberg, only. It is well known that Shubin proved that almost periodic pseudodifferential operators act continuously on the space of smooth almost periodic functions as well as that the operator norm on $L^{2}$ equals that on the Hilbert space $B^{2}\left(\mathbb{R}^{n}\right)$ of Besicovitch almost periodic functions whose Fourier coefficients are square summable. It is also well known that Shubin introduced, for every exponent $p \in[1, \infty]$ and for every real number $t \in \mathbb{R}$, the space $W_{t}^{p}\left(\mathbb{R}^{n}\right)$ of almost periodic functions and proved the continuity of any almost periodic pseudo-differential operator $A: W_{t}^{2}\left(\mathbb{R}^{n}\right) \longrightarrow$ $W_{t-m}^{2}\left(\mathbb{R}^{n}\right)$, with arbitrary $t \in \mathbb{R}$, provided that the symbol of $A$ belongs to the class $S_{\rho, \delta}^{m}(0 \leq \delta<\rho \leq 1)$. In the papers of Shubin, some regularity results for formally hypoelliptic almost periodic pseudo-differential operators have been examined on the space $W_{-\infty}^{2}\left(\mathbb{R}^{n}\right):=\cup_{t \in \mathbb{R}} W_{t}^{2}\left(\mathbb{R}^{n}\right)$.

In [215], the authors sought for ultradistributional analogues of the aforementioned results, working with almost periodic functions that are Gevrey regular of order $s \geq 1$ (the difference between the real analytic case $s=1$ and the pure ultradistributional case $s>1$ should be emphasized here). If $\varnothing \neq \Omega \subseteq \mathbb{R}^{n}$, then the space of all Gevrey functions of order $s \geq 1$, denoted by $G^{s}(\Omega)$, is defined as a collection of all infinitely differentiable functions $F: \mathbb{R}^{n} \longrightarrow \mathbb{C}$ such that, for each compact set $K \subseteq \mathbb{R}^{n}$, there exists a finite real constant $C_{K}>0$ such that $\left|D^{\alpha} F(\mathbf{t})\right| \leq C_{K}^{1+|\alpha|} \alpha !^{s}$ for all $\mathbf{t} \in K$ and $\alpha \in \mathbb{N}_{0}^{n}$. It is natural to ask whether an almost periodic function $F: \mathbb{R}^{n} \longrightarrow \mathbb{C}$ which belongs to the space $G^{s}(\Omega)$ obeys the property of the existence of a global real constant $C>0$ such that $\left|D^{\alpha} F(\mathbf{t})\right| \leq C^{1+|\alpha|} \alpha !^{s}$ for all $\mathbf{t} \in \mathbb{R}^{n}$ and $\alpha \in \mathbb{N}_{0}^{n}$. An instructive counterexample in the one-dimensional setting, with $s>1$, is given in [215], Example 2.1, showing that this is not true in general: set $g_{s}(x):=\exp \left(-x^{(1 /(1-s))}\right), x>0$, $g_{s}(x):=0, \quad x \leq 0, \quad \psi_{s}(x):=g_{s}(x) g_{s}(1-x), \quad x \in \mathbb{R}$, $\psi_{s, n}(x):=\psi_{s}(n x), \quad x \in \mathbb{R}, \quad$ and $\quad \varphi_{s, n}(x):=$ $\sum_{k \in \mathbb{Z}} \psi_{s}\left(x-2^{n}(2 k+1)\right), x \in \mathbb{R}(n \in \mathbb{N})$. It has been shown that the function

$$
F_{s}(x):=\sum_{n=1}^{\infty} n^{-(1 / 4)} \varphi_{s, n}(x), \quad x \in \mathbb{R},
$$

is well defined, as well as that the above series is uniformly convergent in the variable $x \in \mathbb{R}$, so that the function $F_{s}(\cdot)$ is actually semiperiodic since the function $\varphi_{s, n}(\cdot)$ is of period $2^{n+1}(n \in \mathbb{N})$. We also have that $F_{s} \in G^{s}(\mathbb{R})$ as well as that $F_{s} \notin G_{a p}^{s}(\mathbb{R})$; see the notion explained in the following. Albeit not explicitly constructed in [215], it is our strong belief that this example can be transferred to the multidimensional setting without any serious difficulties, as well (more to the point, the case $s=1$ has not been considered in [215], Example 2.1, and deserves further analyses).

After providing this counterexample, the authors paid special attention to the analysis of almost periodic functions $F: \mathbb{R}^{n} \longrightarrow \mathbb{C}$ belonging to the space $G^{s}\left(\mathbb{R}^{n}\right)$ and obeying the property that there exists a real constant $C>0$ such that $\left|D^{\alpha} F(\mathbf{t})\right| \leq C^{1+|\alpha|} \alpha !^{s}$ for all $\mathbf{t} \in \mathbb{R}^{n}$ and $\alpha \in \mathbb{N}_{0}^{n}$. The union of these functions, denoted by $G_{a p}^{s}\left(\mathbb{R}^{n}\right)$, is equipped with the usual inductive limit topology as a union of Banach spaces. Then, the authors introduced the corresponding classes of symbols and pseudo-differential operators and continued their nontrivial analysis; see [215] for more details.

The theory of almost periodic-type functions is far from being completed, and finally, we would like to mention some topics that are not very well explored in the existing literature by now:

(1) Almost anything has been said about the almost periodic properties and the almost automorphic properties of various types of fractional integrals and fractional derivatives of vector-valued periodic functions (see Area, Losada, and Nieto [216] and Jonnalagadda [217]).

(2) The notion of $c$-periodicity and the notion of $c$-almost periodicity require several further investigations within the theory of vector-valued generalized functions [218].

(3) Applications of the multidimensional almost periodic-type functions in the classical theory of partial differential equations and applications of the multidimensional almost periodic-type functions to the boundary value problems are still not examined to a satisfactory extent.

(4) The Stepanov, Weyl, and Besicovitch classes of multidimensional almost automorphic functions have not been analyzed before. See also the recent research studies [219, 220, 221].

(5) The results about the invariance of certain kinds of generalized almost periodicity and generalized almost automorphicity under the actions of infinite convolution products (11) and (12) can be simply transferred to the multidimensional setting. It is not clear how we can apply these results in mathematical physics and applied science.

\section{Conclusions}

In this survey article, we have collected several known results about vector-valued almost periodic functions, separately considering the almost periodic functions of one real variable and the almost periodic functions of several real variables. We have tried to present the most representative applications of almost periodic functions to the abstract Volterra integrodifferential equations in Banach spaces as well as to remind our readers of some landmarks, pioneering investigations of almost periodic functions. We have proposed some open problems and perspectives for further investigations of almost periodicity. 


\section{Data Availability}

The data used to support the findings of this study are available from the corresponding author upon request.

\section{Conflicts of Interest}

The authors declare that there are no conflicts of interest regarding the publication of this paper.

\section{Acknowledgments}

Wei-Shih Du is partially supported by Grant No. MOST 1092115-M-017-002 of the Ministry of Science and Technology of the Republic of China. Marko Kostic is partially supported by the Ministry of Science and Technological Development, Republic of Serbia (grant no. 451-03-68/2020/14/200156). Manuel Pinto is partially supported by FONDECYT (1170466).

\section{References}

[1] H. Bohr, "Zur theorie der fastperiodischen Funktionen I; II; III," Acta Mathematica, vol. 45, pp. 29-127, 1924.

[2] M. Levitan, Almost Periodic Functions, Springer, Berlin, Germany, in Russian, 1953.

[3] S. Bochner, "A new approach to almost periodicity," Proceedings of the National Academy of Sciences, vol. 48, no. 12, pp. 2039-2043, 1962.

[4] G. M. N. Guérékata, Almost Automorphic and Almost Periodic Functions in Abstract Spaces, Kluwer, Dordrecht, The Netherlands, 2001.

[5] M. Kostić, Selected Topics in Almost Periodicity, De Gruyter, Berlin, Germany, 2021.

[6] H. Bart and S. Goldberg, "Characterizations of almost periodic strongly continuous groups and semigroups," Mathematische Annalen, vol. 236, no. 2, pp. 105-116, 1978.

[7] C. Foias and S. Zaidman, "Almost-periodic solutions of parabolic systems," Annali della Scuola normale superiore di Pisa, vol. 15, pp. 247-262, 1961.

[8] V. V. Zhikov, "On the question of harmonic analysis of bounded solutions of operator equations," Doklady Akademii Nauk, vol. 169, pp. 1254-1257, 1966.

[9] V. V. Zhikov, "On a problem of Bochner and von Neumann," Mathematical Notes, vol. 3, pp. 529-538, 1968.

[10] A. I. Perov and T. K. Hai, "Almost periodic solutions of s homogeneous differential equations in a Banach space," Differential Equations, vol. 8, pp. 453-458, 1972.

[11] V. Q. Phóng, Almost Periodic and Stable Semigroups of Operators, Banach Center Publications, Amsterdam, Netherlands, 1997.

[12] M. Kostić, Almost Periodic and Almost Automorphic Type Solutions to Integro-Differential Equations, de Gruyter, Berlin, Germany, 2019.

[13] V. Q. Phóng and Y. I. Lyubich, “A spectral criterion for asymptotic almost periodicity of uniformly continuous representations of abelian semigroups," Journal of Soviet Mathematics, vol. 51, pp. 1263-1266, 1990.

[14] V. Q. Phóng and Y. I. Lyubich, "A spectral criterion for almost periodicity of one-parameter semigroups," Journal of Soviet mathematics, vol. 48, pp. 644-647, 1990.
[15] W. M. Ruess and W. H. Summers, "Asymptotic almost periodicity and motions of semigroups of operators," Linear Algebra and Its Applications, vol. 84, pp. 335-351, 1986.

[16] W. M. Ruess and W. H. Summers, "Compactness in spaces of vector valued continuous functions and asymptotic almost periodicity," Mathematische Nachrichten, vol. 135, no. 1, pp. 7-33, 1988.

[17] W. M. Ruess and W. H. Summers, "Integration of asymptotically almost periodic functions and weak asymptotic almost periodicity," Dissertationes Mathematicae, vol. 279, p. 35, 1989.

[18] W. M. Ruess and W. H. Summers, "Weak almost periodicity and the strong ergodic limit theorem for periodic evolution systems," Journal of Functional Analysis, vol. 94, no. 1, pp. 177-195, 1990.

[19] H. R. Henríquez, "On Stepanov-almost periodic semigroups and cosine functions of operators," Journal of Mathematical Analysis and Applications, vol. 146, no. 2, pp. 420-433, 1990.

[20] M. Bahaj and O. Sidki, "Almost periodic solutions of semilinear equations with analytic semigroups in Banach spaces," Electronic Journal of Differential Equations, vol. 98, pp. 1-11, 2002.

[21] T. A. Burton, Stability and Periodic Solutions of Ordinary and Functional Differential Equations, Academic Press, Orlando, FL, USA, 1985.

[22] J. H. Liu, G. M. N. Guerekata, and N. V. Minh, Topics on Stability and Periodicity in Abstract Differential Equations, Series on Concrete and Applicable Mathematics, Singapore, 2008.

[23] T. Yoshizawa, "Stability theory and the existence of periodic solutions and almost periodic solutions." in Applied Mathematical SciencesSpringer-Verlag, New York, NY, USA, 1975.

[24] I. Cioranescu, "Characterizations of almost periodic strongly continuous cosine operator functions," Journal of Mathematical Analysis and Applications, vol. 116, no. 1, pp. 222-229, 1986.

[25] W. Arendt and C. J. K. Batty, "Almost periodic solutions of first- and second-order Cauchy problems," Journal of Differential Equations, vol. 137, no. 2, pp. 363-383, 1997.

[26] A. S. Avakian, "Almost periodic functions and the vibrating membrane," Journal of Mathematics and Physics, vol. 14, no. 1-4, pp. 350-378, 1935.

[27] M. Ayachi, J. Blot, and P. Cieutat, "Almost periodic solutions of monotone second-order differential equations," Advanced Nonlinear Studies, vol. 11, pp. 541-555, 2011.

[28] Y. M. Berezansky, "On generalized almost periodic functions and sequences, related with the difference-differential equations," Matematicheskii Sbornik, vol. 32, pp. 157-194, 1953, (in Russian).

[29] H. R. Henríquez, M. Pierri, and V. Rolnik, "Pseudo $\mathrm{S}$-asymptotically periodic solutions of second-order abstract Cauchy problems," Applied Mathematics and Computation, vol. 274, pp. 590-603, 2016.

[30] A. S. Rao, "On the Stepanov almost periodic solution of a second-order infinitesimal generator differential equation," International Journal of Mathematics and Mathematical Sciences, vol. 14, no. 4, pp. 757-761, 1991.

[31] S. L. Sobolev, "On almost periodicity for solutions of a wave equation. I-III,” Doklady Akademii Nauk, vol. 48, pp. 570-573, 1945.

[32] R. Yuan, "Existence of almost periodic solutions of second order neutral delay differential equations with piecewise constant argument," Science in China Series A: Mathematics, vol. 41, no. 3, pp. 232-241, 1998. 
[33] S. Zaidman, "Spectrum of almost-periodic solutions for some abstract differential equations," Journal of Mathematical Analysis and Applications, vol. 28, no. 2, pp. 336-338, 1969.

[34] T. Diagana, J. H. Hassan, and S. A. Messaoudi, Existence of Asymptotically Almost Periodic Solutions for Some Second-Order Hyperbolic Integrodifferential Equations, Semigroup Forum in press, Berlin, Germany, 2021.

[35] J. Prüss, Evolutionary Integral Equations and Applications, Birkhäuser-Verlag, Basel, Switzerland, 1993.

[36] Q.-P. Vu, "Almost periodic solutions of Volterra equations," Differential Integral Equations, vol. 7, pp. 1083-1093, 1994.

[37] J. Mu, Y. Zhoa, and L. Peng, "Periodic solutions and Sasymptotically periodic solutions to fractional evolution equations," Discrete Dynamics in Nature and Society, vol. 2017, Article ID 1364532, 12 pages, 2017.

[38] R. P. Agarwal, B. d. Andrade, and C. Cuevas, "On type of periodicity and ergodicity to a class of fractional order differential equations," Advances in Difference Equations, vol. 2010, Article ID 179750, 19 pages, 2010.

[39] P. Bedi, A. Kumar, A. Kumar, T. Abdeljawad, and A. Khan, "S-asymptotically periodic mild solutions and stability analysis of Hilfer fractional evolution equations," Evolution Equations \& Control Theory, 2019, in press.

[40] D. Brindle and G. M. N. '. Guérékata, "S-asymptotically $\omega$ -periodic mild solutions to fractional differential equations," Electronic Journal of Differential Equations, vol. 30, pp. 1-12, 2020.

[41] M. Kostić, Abstract Degenerate Volterra Integro-Differential Equations, Mathematical Institute SANU, Belgrade, Serbia, 2020.

[42] R. Ponce, "Bounded mild solutions to fractional integro-differential equations in Banach spaces," Semigroup Forum, vol. 87, no. 2, pp. 377-392, 2013.

[43] S. Abbas, V. Kavitha, and R. Murugesu, "Stepanov-like weighted pseudo almost automorphic solutions to fractional order abstract integro-differential equations," Proceedings - Mathematical Sciences, vol. 125, no. 3, pp. 323-351, 2015.

[44] Y. Chang and X. Luo, "Pseudo almost automorphic behavior of solutions to a semi-linear fractional differential equation," Mathematical Communications, vol. 20, pp. 53-68, 2015.

[45] A. Debbouche and M. M. El-Borai, "Weak almost periodic and optimal mild solutions of fractional evolution equations," Electronic Journal of Differential Equations, vol. 46, pp. 1-8, 2009.

[46] Q. Li, M. Wei, and M. Wei, "Existence and asymptotic stability of periodic solutions for neutral evolution equations with delay," Evolution Equations \& Control Theory, vol. 9, no. 3, pp. 753-772, 2020.

[47] Y. Min and Q. Wang, "Pseudo asymptotically periodic solutions for fractional integro-differential neutral equations," Science China Math, vol. 62, pp. 1705-1718, 2019.

[48] Z. Xia, "Pseudo asymptotically periodic solutions of two-term time fractional differential equations with delay," Kodai Mathematical Journal, vol. 38, no. 2, pp. 310-332, 2015.

[49] S. Zaidman, "Almost-periodic functions in abstract spaces," in Pitman Research Notes in MathPitman, Boston, MA, USA, 1985.

[50] V. Barbu and A. Favini, "Periodic problems for degenerate differential equations," Rendiconti dell'Istituto di Matematica dell'Università di Trieste, vol. 28, pp. 29-57, 1997.

[51] J. Prüss, "On the spectrum of C 0 -semigroups," Transactions of the American Mathematical Society, vol. 284, no. 2, pp. 847-857, 1984.
[52] C. Lizama and R. Ponce, "Periodic solutions of degenerate differential equations in vector-valued function spaces," Studia Mathematica, vol. 202, no. 1, pp. 49-63, 2011.

[53] B. I. Ptashnic, Ill-Posed Boundary Value Problems for Partial Differential Equations, Naukova Dumka, Kiev, Ukraina, in Russian, 1984.

[54] O. Vejvoda, "Periodic solutions of a linear and weakly nonlinear wave equation in one dimension, I," Czechoslovak Mathematical Journal, vol. 14, no. 3, pp. 341-382, 1964.

[55] O. Vejvoda, L. Herrmann, V. Lovicar, and contributors), Partial Differential Equations: Time-Periodic Solutions, Martinus Nijhoff Publishers, The Hague, Netherlands, 1981.

[56] L. Berselli and L. Bisconti, "On the existence of almost-periodic solutions for the 2D dissipative Euler equations," Revista Matemática Iberoamericana, vol. 31, no. 1, pp. 267-290, 2015.

[57] L. C. Berselli and M. Romito, "On Leray's problem for almost periodic flows," Journal of Mathematical Sciences, the University of Tokyo, vol. 19, pp. 69-130, 2012.

[58] E. V. Vetchanin, E. A. Mikishanina, and E. A. Mikishanina, "Vibrational stability of periodic solutions of the Liouville equations," Nelineinaya Dinamika, vol. 15, no. 3, pp. 351-363, 2019.

[59] A. D. Myshkis, "On certain problems in the theory of differential equations with deviating argument," Russian Mathematical Surveys, vol. 32, no. 2, pp. 181-213, 1977.

[60] W. Dimbour and V. Valmorin, "Asymptotically antiperiodic solutions for a nonlinear differential equation with piecewise constant argument in a Banach space," Applied Mathematics, vol. 07, no. 15, pp. 1726-1733, 2016.

[61] M. Kostić and D. Velinov, "Asymptotically Bloch-periodic solutions of abstract fractional nonlinear differential inclusions with piecewise constant argument," Functional Analysis and Its Applications, vol. 9, pp. 27-36, 2017.

[62] A. Chávez, S. Castillo, and M. Pinto, "Discontinuous almost periodic type functions, almost automorphy of solutions of differential equations with discontinuous delay and applications," Electronic Journal of Qualitative Theory of Differential Equations, vol. 75, no. 75, pp. 1-17, 2014.

[63] R. Yuan and J. Hong, "The existence of almost periodic solutions for a class of differential equations with piecewise constant argument," Nonlinear Anal, vol. 28, pp. 1439-1450, 1997.

[64] K. L. Cooke and J. Wiener, "Retarded differential equations with piecewise constant delays," Journal of Mathematical Analysis and Applications, vol. 99, no. 1, pp. 265-297, 1984.

[65] S. M. Shah and J. Wiener, "Advanced differential equations with piecewise constant argument deviations," International Journal of Mathematics and Mathematical Sciences, vol. 6, no. 4, pp. 671-703, 1983.

[66] J. Wiener, Generalized Solutions of Functional Differential Equations, World Scientific, Singapore, 1993.

[67] E. Ait Dads and L. Lhachimi, "Pseudo almost periodic solutions for equation with piecewise constant argument," Journal of Mathematical Analysis and Applications, vol. 371, no. 2, pp. 842-854, 2010.

[68] K.-S. Chiu and M. Pinto, "Periodic solutions of differential equations with a general piecewise constant argument and applications," Electronic Journal of Qualitative Theory of Differential Equations, vol. 46, no. 46, pp. 1-19, 2010.

[69] K.-S. Chiu, M. Pinto, and J.-C. Jeng, "Existence and global convergence of periodic solutions in recurrent neural network models with a general piecewise alternately advanced and retarded argument," Acta Applicandae Mathematicae, vol. 133, no. 1, pp. 133-152, 2014. 
[70] M. I. Muminov, "On the method of finding periodic solutions of second-order neutral differential equations with piecewise constant arguments," Advances in Difference Equations, vol. 336, 2017.

[71] G. Papaschinopoulos, "Some results concerning a class of differential equations with piecewise constant argument," Mathematische Nachrichten, vol. 166, no. 1, pp. 193-206, 1994.

[72] M. Pinto, "Cauchy and Green matrices type and stability in alternately advanced and delayed differential systems," Journal of Difference Equations and Applications, vol. 17, no. 2, pp. 235-254, 2011.

[73] R. Yuan, "The existence of almost periodic solutions of retarded differential equations with piecewise constant argument," Nonlinear Analysis: Theory, Methods \& Applications, vol. 48, no. 7, pp. 1013-1032, 2002.

[74] G. M. N. Guérékata and M. Kostić, "Generalized asymptotically almost periodic and generalized asymptotically almost automorphic solutions of abstract multi-term fractional differential inclusions," Abstract and Applied Analysis, vol. 2018, Article ID 5947393, 17 pages, 2018.

[75] T. Diagana, Almost Automorphic Type and Almost Periodic Type Functions in Abstract Spaces, Springer, Berlin, Germany, 2013.

[76] L. Amerio and G. Prouse, Almost Periodic Functions and Functional Equations, Van Nostrand-Reinhold, New York, NY, 1971.

[77] L. N. Argabright and J. G. de Lamadrid, "Almost periodic measures," Memoirs of the American Mathematical Society, vol. $428,1990$.

[78] M. Baake and U. Grimm, "Aperiodic order," in A Mathematical InvitationCambridge University Press, Cambridge, UK, 2013.

[79] M. Baake and U. Grimm, "Aperiodic order," in Crystallography and Almost PeriodicityCambridge University Press, Cambridge, UK, 2017.

[80] P. H. Bezandry and T. Diagana, Almost Periodic Stochastic Processes, Springer, Berin, Germany, 2011.

[81] A. Böttcher, I. Y. Karlovich, and I. M. Spitkovsky, Convolution Operators and Factorization of Almost Periodic Matrix Functions, Birkhäuser-Verlag, Basel, Switzerland, 2002.

[82] A. Böttcher, "On the corona theorem for almost periodic functions," Integral Equations Operator Theory, vol. 33, pp. 253-272, 1999.

[83] P. Boggiatto, C. Fernández, and A. Galbis, "Gabor systems and almost periodic functions," Applied and Computational Harmonic Analysis, vol. 42, no. 1, pp. 65-87, 2017.

[84] Y. H. Kim, "Representations of almost-periodic functions using generalized shift-invariant systems in $R^{d}$, Journal of Fourier Analysis and Applications, vol. 19, no. 4, pp. 857-876, 2013.

[85] Y.-K. Chang, G. M. N. Guerekata, and R. Ponce, "Bloch-type periodic functions: theory and applications to evolution equations," 2021.

[86] D. N. Cheban, Asymptotically Almost Periodic Solutions of Differential Equations, Hindawi Publishing Corporation, London, UK, 2009.

[87] E. Yu. Emel'yanov, Non-Spectral Asymptotic Analysis of One-Parameter Operator Semigroups, Birkhäuser-Verlag, Basel, Switzerland, 2007.

[88] Y. Hino, T. Naito, N. V. Minh, and J. S. Shin, "Almost periodic solutions of differential equations in Banach spaces," Stability and Control: Theory, Methods and Applications, vol. 15, 2002.
[89] G. M. N. '. Guérékata, Spectral Theory of Bounded Functions and Applications to Evolution Equations, Nova Science Publishers, New York, NY, USA, 2017.

[90] J. L. Massera, "The existence of periodic solutions of systems of differential equations," Duke Mathematical Journal, vol. 17, pp. 457-475, 1950.

[91] R. Hsu, Topics on Weakly Almost Periodic Functions, State University of New York at Buffalo, New York, NY, USA, 1985.

[92] G. T. Stamov, Almost Periodic Solutions of Impulsive Differential Equations, Springer-Verlag, Berlin. Germany, 2012.

[93] D. Bainov and P. Simeonov, "Impulsive differential equations: periodic solutions and applications," Pitman Monographs and Surveys in Pure and Applied Mathematics; Longman Scientific and Technical,vol. 66, 1993.

[94] N. A. Perestyuk, V. A. Plotnikov, A. M. Somoilenko, and N. V. Skripnik, Differential Equations with Impulsive Effects. Multivalued Right-Hand Sides with Discontinuities, De Gruyter, Berlin, Germany, 2011.

[95] I. Stamova and G. Stamov, Applied Impulsive Mathematical Models, Springer International Publishing, Berlin, Germany, 2016.

[96] X. Song, H. Gno, and X. Shi, Theory and Applications of Impulsive Differential Equations, Science Press, Beijing, China, 2011.

[97] P. Acquistapace, "Evolution operators and strong solutions of abstract linear parabolic equations," Differential Integral Equations, vol. 1, pp. 433-457, 1988.

[98] P. Acquistapace and B. Terreni, "A uniffied approach to abstract linear nonautonomous parabolic equations," Rendiconti del Seminario Matematico della Università di Padova, vol. 78, pp. 47-107, 1987.

[99] Y.-H. Chang and J.-S. Chen, "The almost periodic solutions of nonautonomous abstract differential equations," Chinese Journal of Mathematics, vol. 23, pp. 257-274, 1995.

[100] K. Khalil, "On the almost periodicity of nonautonomous evolution equations and application to lotka-voltera systems," 2020, https://arxiv.org/abs/2007.01143.

[101] R. Schnaubelt, "A sufficient condition for exponential dichotomy of parabolic evolution equations," in Evolution Equations and Their Applications in Physical and Life Sciences (Proceedings Bad Herrenalb, 1998), G. Lumer and L. Weis, Eds., pp. 149-158, Marcel Dekker, New York, NY, USA, 2000.

[102] V. V. Zhikov, "Abstract equations with almost-periodic coefficients,” Doklady Akademii Nauk, vol. 163, pp. 555-558, 1965.

[103] V. V. Zhikov, "Almost periodic solutions of differential equations in Hilbert space," Doklady Akademii Nauk, vol. 165 , pp. 1227-1230, 1965.

[104] M. Baroun, L. Maniar, and R. Schnaubelt, "Almost periodicity of parabolic evolution equations with inhomogeneous boundary values," Integral Equations and Operator Theory, vol. 65, no. 2, p. 169, 2009.

[105] M. Baroun, K. Ezzinbi, K. Khalil, and L. Maniar, "Almost automorphic solutions for nonautonomous parabolic evolution equations," Semigroup Forum, vol. 99, no. 3, pp. 525-567, 2019.

[106] D. A. Zakora, "Abstract linear Volterra second-order integrodifferential equations," Eurasian Mathematical Journal, vol. 7, pp. 75-91, 2016.

[107] M. Bochner and A. Peterson, Dynamic Equations on Time Scales. An Introduction with Applications, Birkhäuser Boston, Boston, MA, USA, 2001. 
[108] E. Alvarez, S. Castillo, and M. Pinto, “ $(\omega, c)$-Pseudo periodic functions, first order Cauchy problem and Lasota-Wazewska model with ergodic and unbounded oscillating production of red cells. Bound," Value Problems, vol. 106, pp. 1-20, 2019.

[109] E. Alvarez, A. Gómez, and M. Pinto, " $(\omega, c)$-periodic functions and mild solutions to abstract fractional integro-differential equations," Electronic Journal of Qualitative Theory of Differential Equations, vol. 16, no. 16, pp. 1-8, 2018.

[110] E. Alvarez, S. Castillo, and M. Pinto, " $(\omega, c)$-asymptotically periodic functions, first-order Cauchy problem, and Lasota-Wazewska model with unbounded oscillating production of red cells," Mathematical Methods in the Applied Sciences, vol. 43, no. 1, pp. 305-319, 2020.

[111] M. Wazewska-Czyzewska and A. Lasota, "Mathematical problems of the red-blood cell system," Applied Mathematics, vol. 6, pp. 23-40, 1976.

[112] J. R. Wang, L. Ren, and Y. Zhou, "Periodic solutions for time varying impulsive differential equations," Advances in Difference Equations, vol. 259, 2019.

[113] G. Mophou, G. M. N. Guérékata, and A. Milce, "An Existence Result of,periodic mild solutions to some fractional differential equation," Nonlinear Studies, vol. 27. , 2020 In press.

[114] M. Li, J.-R. Wang, and M. Fečkan, "Periodic solutions for impulsive differential systems," Communications in Mathematical Analysis, vol. 21, pp. 35-45, 2018.

[115] E. Ait Dads, K. Ezzinbi, and O. Arino, "Pseudo almost periodic solutions for some differential equations in a Banach space," Nonlinear Anal, vol. 28, pp. 1145-1155, 1997.

[116] J. Blot, P. Cieutat, and K. Ezzinbi, "New approach for weighted pseudo-almost periodic functions under the light of measure theory, basic results and applications," Applicable Analysis, vol. 65, pp. 1-34, 2011.

[117] J. Blot, P. Cieutat, and K. Ezzinbi, "Measure theory and pseudo almost automorphic functions: new developments and applications," Nonlinear Analysis: Theory, Methods \& Applications, vol. 75, no. 4, pp. 2426-2447, 2012.

[118] A. Coronel, M. Pinto, and D. Sepúlveda, "Weighted pseudo almost periodic functions, convolutions and abstract integral equations," Journal of Mathematical Analysis and Applications, vol. 435, no. 2, pp. 1382-1399, 2016.

[119] T. Diagana, Pseudo Almost Periodic Functions in Banach Spaces, Nova Science Publishers, New York, NY, USA, 2007.

[120] T. Diagana, "Weighted pseudo almost periodic functions and applications," Comptes Rendus Mathematique, vol. 343, no. 10 , pp. 643-646, 2006.

[121] T. Diagana, "Existence of almost periodic solutions to some third-order nonautonomous differential equations," Electronic Journal of Qualitative Theory of Differential Equations, vol. 28, no. 65, pp. 1-14, 2011.

[122] T. Diagana, "Existence of doubly-weighted pseudo almost periodic solutions to non-autonomous differential equations," African Diaspora Journal of Mathematic, vol. 12, pp. 121-136, 2011.

[123] D. Ji and C. Zhang, "Translation invariance of weighted pseudo almost periodic functions and related problems," Journal of Mathematical Analysis and Applications, vol. 391, no. 2, pp. 350-362, 2012.

[124] J. Liang, T.-J. Xiao, and J. Zhang, "Decomposition of weighted pseudo-almost periodic functions," Nonlinear Analysis: Theory, Methods \& Applications, vol. 73, no. 10, pp. 3456-3461, 2010.

[125] C. Zhang, Pseudo almost periodic functions and their applications, PhD. Thesis, The University of Western Ontario, London, UK, 1992.
[126] C. Y. Zhang, "Pseudo almost periodic solutions of some differential equations," Journal of Mathematical Analysis and Applications, vol. 181, no. 1, pp. 62-76, 1994.

[127] C. Y. Zhang, "Pseudo almost periodic solutions of some differential equations, II," Journal of Mathematical Analysis and Applications, vol. 192, no. 2, pp. 543-561, 1995.

[128] J. Zhang, T.-J. Xiao, and J. Liang, "Weighted pseudo almost periodic functions and applications to semilinear evolution equations," Abstract and Applied Analysis, vol. 2012, Article ID 179525, 15 pages, 2012.

[129] L. Zhang and Y. Xu, "Weighted pseudo-almost periodic solutions of a class of abstract differential equations," Nonlinear Analysis: Theory, Methods \& Applications, vol. 71, no. 9, pp. 3705-3714, 2009.

[130] L.-L. Zhang and H.-X. Li, "Weighted pseudo-almost periodic solutions for some abstract differential equations with uniform continuity," Bulletin of the Australian Mathematical Society, vol. 82, no. 3, pp. 424-436, 2010.

[131] S. J. Favorov, "Zeros of holomorphic almost periodic functions," Journal d'Analyse Mathématique, vol. 84, no. 1, pp. 51-66, 2001.

[132] B. Jessen, "Some aspects of the theory of almost periodic functions," in Proceedings of the . Internat. Congress Mathematicians, pp. 304-351, Amsterdam, Netherlands, September 1954.

[133] J. M. Sepulcre and T. Vidal, "Almost periodic functions in terms of Bohr's equivalence relation," The Ramanujan Journal, vol. 46, no. 1, pp. 245-267, 2018.

[134] J. M. Sepulcre and T. Vidal, "Sets of values of equivalent almost periodic functions," The Ramanujan Journal, 2021, In press.

[135] V. Borchsenius and B. Jessen, "Mean motions and values of the Riemann zeta function," Acta Mathematica, vol. 80, pp. 97-166, 1948.

[136] G. Mora and J. M. Sepulcre, "On the distribution of zeros of a sequence of entire functions approaching the Riemann zeta function," Journal of Mathematical Analysis and Applications, vol. 350, no. 1, pp. 409-415, 2009.

[137] S. Favorov and O. Udodova, "Almost periodic functions in finite-dimensional space with the spectrum in a cone. preprint," 2019, https://arxiv.org/abs/astro-ph/0701820.

[138] S. Y. Favorov and A. V. Rakhnin, "Subharmonic almost periodic functions," Journal of Mathematical Physics, Analysis, Geometry.vol. 2, pp. 209-224, 2005.

[139] S. Yu. Favorov and A. V. Rakhnin, "Subharmonic almost periodic functions of slowth growth," Journal of Mathematical Physics, Analysis, Geometry, vol. 1, pp. 109-127, 2007.

[140] S. Y. Favorov and A. Y. Rashkovskii, "Holomorphic almost periodic functions," Acta Applicandae Mathematicae, vol. 65, no. 1/3, pp. 217-235, 2001.

[141] M. Adamczak, " $C^{(n)}$-almost periodic functions," Commentationes Mathematicae (Prace Matematyczne), vol. 37, pp. 1-12, 1997.

[142] T. Diagana, V. Nelson, and G. M. N. Guérékata, "Almost automorphic mild solutions to some classes of nonautonomous higher-order differential equations," Semigroup Forum, vol. 82, no. 3, pp. 455-477, 2011.

[143] M. Kostić, "Weyl-almost periodic solutions and asymptotically Weyl-almost periodic solutions of abstract Volterra integro-differential equations," Banach Journal of Mathematical Analysis, vol. 13, pp. 64-90, 2019.

[144] M. Kostić, "Asymptotically Weyl almost periodic functions in Lebesgue spaces with variable exponents," Journal of 
Mathematical Analysis and Applications, vol. 498, no. 1, Article ID 124961, 2021, In press.

[145] M. Kostić, "Multi-dimensional -almost Periodic Type Functions and Applications," 2020.

[146] M. Kostić and W.-S. Du, "Generalized almost periodicity in Lebesgue spaces with variable exponents," Mathematics, vol. 8 , no. 6 , p. 928,2020 .

[147] M. Kostić and W.-S. Du, "Generalized almost periodicity in Lebesgue spaces with variable exponents, Part II," Mathematics, vol. 8, no. 7, p. 1052, 2020.

[148] R. Lucchetti and F. Patrone, "On Nemytskii's operator and its application to the lower semicontinuity of integral functionals," Indiana University Mathematics Journal, vol. 29, no. 5, pp. 703-713, 1980.

[149] J. Blot, P. Cieutat, G. M. N. Guérékata, and D. Pennequin, "Superposition operators between various almost periodic function spaces and applications," Communications in Mathematical Analysis, vol. 6, pp. 42-70, 2009.

[150] P. Cieutat, "Nemytskii operators between stepanov almost periodic or almost automorphic function spaces," 2019, https://arxiv.org/abs/1910.09389.

[151] T. R. Hillmann, "Besicovitch-Orlicz spaces of almost periodic functions," in Real and Stochastic AnalysisWiley, Hoboken , NJ, USA, 1986.

[152] M. Morsli and M. Smaali, "Characterization of the strict convexity of the Besicovitch-Musielak-Orlicz space of almost periodic functions," Commentationes Mathematicae Universitatis Carolinae, vol. 48, pp. 443-458, 2007.

[153] F. Bedouhene, Y. Djabri, and F. Boulahia, "Ergodicity in stepanov-orlicz spaces," Annals of Functional Analysis, vol. 11, pp. 1-17, 2019.

[154] A. Haraux and V. Komornik, "Density theorems for almost periodic functions: a Hilbert space approach," Journal of Mathematical Analysis and Applications, vol. 122, pp. 538554, 1987.

[155] M. Akhmet, "Almost periodicity, chaos, and asymptotic equivalence," Nonlinear Systems and Complexity, vol. 27, 2020.

[156] M. Kostić, Chaos for Linear Operators and Abstract Differential Equations, Nova Science Publishers, New York, NY, USA, 2020.

[157] S. Abbas, S. Dhama, M. Pinto, and D. Sepúlveda, "Pseudo compact almost automorphic solutions for a family of delayed population model of Nicholson type," Journal of Mathematical Analysis and Applications, vol. 495, no. 1, Article ID 124722, 2021.

[158] H.-S. Ding, Q.-L. Liu, and J. J. Nieto, "Existence of positive almost periodic solutions to a class of hematopoiesis model," Applied Mathematical Modelling, vol. 40, no. 4, pp. 3289-3297, 2016.

[159] H. Zhang, M. Yang, and L. Wang, "Existence and exponential convergence of the positive almost periodic solution for a model of hematopoiesis," Applied Mathematics Letters, vol. 26, no. 1, pp. 38-42, 2013.

[160] M. Mackey and L. Glass, "Oscillation and chaos in physiological control systems," Science, vol. 197, no. 4300, pp. 287-289, 1977.

[161] J. Mycielski, "On a problem of interpolation by periodic functions," Colloquium Mathematicum, vol. 8, no. 1, pp. 95-97, 1961.

[162] J. S. Lipiński, "Sur un problème de E. Marczewski concernant les fonctions périodiques," Bulletin L'Académie Polonaise des Science, vol. 8, pp. 695-697, 1960, in French.

[163] S. Hartman, "On interpolation by almost periodic functions," Colloquium Mathematicum, vol. 8, no. 1, pp. 99-101, 1961.
[164] S. Hartman and C. Ryll-Nardzewski, "Almost periodic extensions of functions," Colloquium Mathematicum, vol. 12, no. 1, pp. 23-39, 1964.

[165] S. Hartman and C. Ryll-Nardzewski, "Almost periodic extensions of functions, II," Colloquium Mathematicum, vol. 15, no. 1, pp. 79-86, 1966.

[166] S. Hartman and C. Ryll-Nardzewski, "Almost periodic extensions of functions, III," Colloquium Mathematicum, vol. 16, no. 1, pp. 223-224, 1967.

[167] E. Strzelecki, "On a problem of interpolation by periodic and almost periodic functions," Colloquium Mathematicum, vol. 11, no. 1, pp. 91-99, 1963.

[168] S. Hartman, "Remark on interpolation by L-almost periodic functions," Colloquium Mathematicum, vol. 30, no. 1, pp. 133-136, 1974.

[169] A. S. Besicovitch, Almost Periodic Functions, Dover Publications, New York, NY, USA, 1954.

[170] A. A. Pankov, Bounded and Almost Periodic Solutions of Nonlinear Operator Differential Equations, Kluwer Academic Publishers, Dordrecht, Netherlands, 1990.

[171] L. Rodman, I. M. Spitkovsky, and H. J. Woerdeman, "Contractive extension problems for matrix valued almost periodic functions of several variables," Journal of Operator Theory, vol. 47, pp. 3-35, 2002.

[172] L. Rodman and I. M. Spitkovsky, "Algebras of almost periodic functions with Bohr-Fourier spectrum in a semigroup: hermite property and its applications," Journal of Functional Analysis, vol. 255, pp. 3188-3207, 2011.

[173] L. Rodman, I. M. Spitkovsky, and H. J. Woerdeman, "Multiblock problems for almost periodic matrix functions of several variables," New York Journal of Mathematics, vol. 7, pp. 117-148, 2001.

[174] Y. Meyer, "Mean-periodic functions and irregular sampling," Transactions of the Royal Society, vol. 1, pp. 5-23, 2018.

[175] J.-P. Kahane, Lectures on Mean Periodic Functions, Tata Institute of Fundamental Research, Bombay, India, 1959.

[176] R. B. Basit, "Generalization of two theorems of M. I. Kadets concerning the indefinite integral of abstract almost periodic functions," Mathematical Notes of the Academy of Sciences of the USSR, vol. 9, no. 3, pp. 181-186, 1971.

[177] S. M. A. Alsulami, "On the integral of almost periodic functions of several variables," Applied Mathematical Sciences, vol. 6, pp. 3615-3622, 2012.

[178] Y. K. Khasanov, "On approximation of almost periodic functions of two real variables," Russian Mathematics (Izvestiya VUZ. Matematika), vol. 12, pp. 82-86, 2010, in Russian.

[179] Y. K. Khasanov, "Absolute convergence of Fourier series of almost-periodic functions," Mathematical Notes, vol. 94, no. 5-6, pp. 692-702, 2013.

[180] Y. K. Khasanov, "On deviation of harmonic almost periodic functions from their boundary values," Vladikavkaz Mathematical Journal, vol. 17, pp. 80-85, 2015.

[181] Y. K. Khasanov, "On absolute Cesáro summablity of Fourier series for almost-periodic functions with limiting points at zero," Ufa Mathematical Journal, vol. 8, pp. 147-155, 2016, in Russian.

[182] Y. K. Khasanov, "On approximation of almost periodic functions by some sums," Vladikavkaz Mathematical Journal, vol. 19, pp. 76-85, 2017, in Russian.

[183] Y. Khasanov, E. Safarzoda, and E. Safarzoda, "On approximation of Stepanov's almost periodic functions by means of Marcinkiewicz," Vestnik Volgogradskogo Gosudarstvennogo 
Universiteta. Serija 1. Mathematica. Physica, vol. 6, no. 6, pp. 61-69, 2016, in Russian.

[184] M. A. Latif and M. I. Bhatti, "Almost periodic functions defined on $!^{n}$ with values in fuzzy setting," Punjab University Journal of Mathematics (Lahore), vol. 39, pp. 19-27, 2007.

[185] M. A. Latif and M. I. Bhatti, "Almost periodic functions defined on !n with values in locally convex spaces," Journal of Prime Research in Mathematics, vol. 4, pp. 181-194, 2008.

[186] G. M. N. Guérékata, M. A. Latif, and M. I. Bhatti, "Almost periodic functions defined $\mathbb{R}^{n}$ on with values in $p$ - Fréchet spaces, $0<p<1$," Libertas Math, vol. 29, pp. 83-100, 2009.

[187] G. R. Sell, "Almost periodic solutions of linear partial differential equations," Journal of Mathematical Analysis and Applications, vol. 42, no. 2, pp. 302-312, 1973.

[188] G. R. Sell, "A note on almost periodic solutions of linear partial differential equations," Bulletin of the American Mathematical Society, vol. 79, no. 2, pp. 428-431, 1973.

[189] A. M. Fink, Almost Periodic Differential Equations, Springer, Berlin, Germany, 1974.

[190] L. Bao-Ping and C. V. Pao, "Almost periodic plane wave solutions for reaction diffusion equations," Journal of Mathematical Analysis and Applications, vol. 105, no. 1, pp. 231-249, 1985.

[191] S. M. A. Alsulami, On evolution equations in banach spaces and commuting semigroups, $\mathrm{PhD}$. Thesis, Ohio University, Athens, OH, USA, 2005.

[192] G. Spradlin, "An almost periodic function of several variables with no local minimum," Rendiconti dell'Istituto di Matematica dell'Università di Trieste, vol. 28, pp. 371-381, 1996.

[193] G. Spradlin, "A singularly perturbed elliptic partial differential equation with an almost periodic term," Calculus of Variations and Partial Differential Equations, vol. 9, pp. 207-232, 1996.

[194] G. Spradlin, "An elliptic partial differential equation with a symmetrical almost periodic term," Calculus of Variations and Partial Differential Equations, vol. 9, pp. 233-247, 1996.

[195] B. I. Ptashnic and P. I. Shtabalyuk, "A boundary value problem for hyperbolic equations in a class of functions that are almost periodic with respect to space variables," Differentsialnye Uravneniya, vol. 22, pp. 669-678, 1986, in Russian.

[196] F. Yang and C. Zhang, "Remotely almost periodic solutions to parabolic inverse problems," Taiwanese Journal of Mathematics, vol. 15, pp. 43-57, 2011.

[197] B. Basit, "Some problems concerning different types of vector valued almost periodic functions," Dissertationes Mathematics, vol. 338, 1995.

[198] B. Dumitrescu, Positive Trigonometric Polynomials and Signal Processing Applications, Springer International Publishing, Berlin, Germany, 2017.

[199] D. Dung, V. Temlyakov, and T. Ullrich, "Hyperbolic cross approximation," in Advanced Courses in MathematicsCRM Barcelona, Basel, Switzerland, 2018.

[200] V. Temlyakov, Approximation of Periodic Functions, Nova Science Publishers, New York, NY, USA, 1993.

[201] V. Temlyakov, Multivariate Approximation, Cambridge University Press, Cambridge, UK, 2018.

[202] V. N. Temlyakov, "Greedy algorithm and m -term trigonometric approximation," Constructive Approximation, vol. 14 , no. 4, pp. 569-587, 1998.

[203] A. M.-B. Babayev, "Approximations of periodic functions of two variables by trigonometric polynomials," Transactions Issue Mathematics, Azerbaijan, vol. 34, pp. 21-28, 2014.
[204] L. Pfister and Y. Bresler, "Bounding multivariate trigonometric polynomials," IEEE Transactions on Signal Processing, vol. 67, no. 3, pp. 700-707, 2019.

[205] L. Kämmerer, D. Potts, and T. Volkmer, “Approximation of multivariate periodic functions by trigonometric polynomials based on rank-1 lattice sampling," Journal of Complexity, vol. 31, no. 4, pp. 543-576, 2015.

[206] L. A. Coburn, R. D. Moyer, and I. M. Singer, "C*-algebras of almost periodic pseudo-differential operators," Acta Mathematica, vol. 130, pp. 279-307, 1973.

[207] P. E. Dedik, "Theorems on the boundedness of almost-periodic pseudodifferential operators," Siberian Mathematical Journal, vol. 22, pp. 361-369, 1981.

[208] R. Iannacci, A. M. Bersani, G. Dell'Acqua, and P. Santucci, "Embedding theorems for Sobolev-Besicovitch spaces of almost periodic functions," Zeitschrift für Analysis und ihre Anwendungen, vol. 17, no. 2, pp. 443-457, 1998.

[209] A. A. Pankov, "Theory of almost-periodic pseudodifferential operators," Ukrainian Mathematical Journal, vol. 33, pp. 469-472, 1981.

[210] M. A. Shubin, "Differential and pseudodifferential operators in spaces of almost periodic functions," Matematicheskii Sbornik, vol. 95, pp. 560-587, 1974.

[211] M. A. Shubin, "Theorems on the coincidence of the spectra of an almost periodic pseudodifferential operator in the spaces $L^{2}\left(!^{n}\right)$ and $B^{2}\left(!^{n}\right)$," Siberian Mathematical Journal, vol. 17 , pp. 200-215, 1976.

[212] M. A. Shubin, "Pseudodifferential almost-periodic operators and von Neumann algebras," Siberian Mathematical Journal, vol. 35, pp. 103-163, 1976.

[213] M. A. Shubin, "Almost periodic functions and partial differential equations," Uspekhi Matematicheskikh Nauk, vol. 33, pp. 3-47, 1978.

[214] P. Wahlberg, "A transformation of almost periodic pseudodifferential operators to Fourier multiplier operators with operator-valued symbols," Rendiconti del Seminario Matematico Università e Politecnico di Torino, vol. 67, pp. 247-269, 2009.

[215] A. Oliaro, L. Rodino, and P. Wahlberg, "Almost periodic pseudodifferential operators and Gevrey classes," Annali di Matematica Pura ed Applicata, vol. 191, no. 4, pp. 725-760, 2012.

[216] I. Area, J. Losada, and J. J. Nieto, "On quasi-periodic properties of fractional sums and fractional differences of periodic functions," Applied Mathematics and Computation, vol. 273, pp. 190-200, 2016.

[217] J. M. Jonnalagadda, "Quasi-periodic solutions of fractional nabla difference systems," Fractional Differential Calculus, vol. 7, no. 2, pp. 339-355, 2017.

[218] M. T. Khalladi, M. Kostić, M. Pinto, A. Rahmani, and D. Velinov, "c-Almost periodic type functions and applications," Nonautonomous Dynamical Systems, vol. 7, no. 1, pp. 176-193, 2020.

[219] A. Chávez, K. Khalil, M. Kostić, and M. Pinto, "Multi-dimensional almost periodic type functions and applications. preprint," 2020, https://arxiv.org/abs/2012.00543.

[220] A. Chávez, K. Khalil, M. Kostić, and M. Pinto, "Stepanov multi-dimensional almost periodic type functions and applications," 2020.

[221] A. Chávez, K. Khalil, M. Kostić, and M. Pinto, "Multi-dimensional almost automorphic type functions and applications," 2021. 